\title{
APC mutant cells exploit compensatory chromosome alterations to restore tumour cell fitness
}

Yoshihiro Kawasaki ${ }^{1,2}$, Tomoko Hamaji ${ }^{1}$, Koji Owada ${ }^{1}$, Akiko Hayashi ${ }^{1}$, Yuping Wu ${ }^{1}$, Taisaku

Nogi $^{1}$, Miwa Okada ${ }^{1}$, Yuta Kouyama ${ }^{3}$, Atsushi Niida ${ }^{4}$, Koshi Mimori ${ }^{3}$, Toshihiko Kuroda ${ }^{5}$, Takao Senda $^{6}$, Miho Ohsugi ${ }^{7}$, Katsumi Fumoto ${ }^{8}$, Akira Kikuchi $^{8}$, Per O. Widlund ${ }^{9}$, Kazuyuki Kiyosue $^{10}$, Norio Yamashita ${ }^{11}$, Masahiko Morita ${ }^{11}$, Hideo Yokota ${ }^{11}$, Satya N. V. Arjunan ${ }^{12}$, WeiXiang Chew ${ }^{12,13}$, Koichi Takahashi ${ }^{12}$, Wesley R. Legant ${ }^{14, \dagger}$, Bi-Chang Chen ${ }^{14, \dagger \dagger}$, Eric Betzig ${ }^{14, \dagger \dagger}$, Ron Smits ${ }^{15}$, Riccardo Fodde ${ }^{16}$, Hiroko Oshima ${ }^{17}$, Masanobu Oshima ${ }^{17}$, M. Mark Taketo ${ }^{18}$, Tetsu Akiyama $^{2}$, Yuko Mimori-Kiyosue ${ }^{1, *}$

${ }^{1}$ Laboratory for Molecular and Cellular Dynamics, RIKEN Center for Biosystems Dynamics Research, Kobe 650-0047, Japan

${ }^{2}$ Laboratory of Molecular and Genetic Information, Institute of Molecular and Cellular Biosciences, The University of Tokyo, Tokyo 113-0032, Japan

${ }^{3}$ Department of Surgery, Kyushu University Beppu Hospital, Beppu 874-0838, Japan

${ }^{4}$ Division of Health Medical Computational Science, Health Intelligence Center, Institute of Medical Science, The University of Tokyo, Tokyo 108-8639, Japan

${ }^{5}$ Nico-tama Coloproctology Clinic, Tokyo 158-0094, Japan

${ }^{6}$ Department of Anatomy, Graduate School of Medicine, Gifu University, Gifu 501-1194, Japan

${ }^{7}$ Department of Life Sciences, Graduate School of Arts and Sciences, The University of Tokyo, Tokyo 153-8902, Japan

${ }^{8}$ Department of Molecular Biology and Biochemistry, Graduate School of Medicine, Osaka University, Osaka 565-0871, Japan

${ }^{9}$ Department of Microbiology and Immunology, University of Gothenburg, Gothenburg 405 30, Sweden

${ }^{10}$ Biomedical Research Institute, National Institute of Advanced Industrial Science and Technology, Ikeda 563-0026, Japan

${ }^{11}$ Image Processing Research Team, Center for Advanced Photonics, RIKEN, Saitama 351-0198, Japan

${ }^{12}$ Laboratory for Biologically Inspired Computing, RIKEN Center for Biosystems Dynamics Research, Osaka 565-0874, Japan

${ }^{13}$ Department of Physics, Faculty of Science, University of Malaya, 50603 Kuala Lumpur, Malaysia 
${ }^{14}$ Janelia Research Campus, Howard Hughes Medical Institute, Ashburn, VA 20147, USA

${ }^{15}$ Department of Gastroenterology and Hepatology, Erasmus MC-University Medical Center, 3015 CE Rotterdam, The Netherlands

${ }^{16}$ Department of Pathology, Erasmus MC Cancer Institute, Erasmus University Medical Center, 3000 CA Rotterdam, The Netherlands

${ }^{17}$ Division of Genetics, Cancer Research Institute, Kanazawa University, Ishikawa 920-1164, Japan

${ }^{18}$ Colon Cancer Project, Kyoto University Hospital-iACT, Graduate School of Medicine, Kyoto University, Yoshida-Honmachi, Sakyo, Kyoto 606-8501

$\dagger$ Joint Department of Biomedical Engineering, UNC, Chapel Hill, NC 27599, USA, and NCSU Raleigh, NC 27695, USA.; Department of Pharmacology, University of North Carolina, Chapel Hill, NC, USA.

${ }^{\dagger}$ Present address: Research Center for Applied Sciences, Academia Sinica, Taipei 115-29, Taiwan

i†Present address: Departments of Physics and Molecular and Cell Biology, University of California, Berkeley, CA 94720, USA.; Howard Hughes Medical Institute, Berkeley, CA 94720, USA.

*Correspondence to: Yuko Mimori-Kiyosue, Ph.D., Laboratory for Molecular and Cellular Dynamics, RIKEN Center for Biosystems Dynamics Research, 2-2-3 Minatojima-minamimachi, Chuo-ku, Kobe 650-0047, Japan.

Tel.: +81-78-306-3070

Fax: +81-78-306-3118

E-mail: yuko.kiyosue@ riken.jp 


\section{Abstract}

Certain copy number alterations (CNAs) are strongly associated with particular cancer types.

However, the mechanisms underlying the selection of specific CNAs remain unknown. Here, we identified functional relationships between recurrent CNAs in colorectal cancers (CRCs) and adenomatous polyposis coli (APC) mutations. Quantitative phenotyping of mitotic spindles highlighted APC functions at centrosomes where APC positively regulated Aurora A kinase (AURKA). Upon APC inactivation, elevated $\beta$-catenin levels blocked AURKA activation, which caused chromosome instability and supressed proliferation, resulting in the generation and selection of AURKA-activating CNAs. Arm-level amplification of chromosomes containing AURKA and AURKA activator genes was observed in $A P C$ mutant CRCs, early stage mouse tumours, and cells in culture, which was concomitant with an increase in growth potential. Our findings demonstrate a mechanism that restores tumour cell fitness through compensatory chromosome alterations to overcome adverse effects of prior mutations, which may affect the course of cancer type-specific CNA formation. 


\section{Introduction}

Chromosome number abnormality is one of the most common characteristics of cancer. Recent advances in genome characterisation technologies have revealed the genomic landscape of cancer and identified recurrent copy number alterations (CNAs) including arm-level and focal CNAs $^{1,2}$. The strong association of certain CNAs with particular cancer types and lineages ${ }^{1,3}$ raises questions regarding the mechanisms underlying the selection of cancer type-specific CNAs and their functional consequences. Additionally, a paradox has emerged: because armlevel CNAs, traditionally aneuploidy ${ }^{4}$, in normal cells exert negative effects on cellular and organismal fitness and growth by disrupting cellular homeostasis ${ }^{4,5}$, it is unclear how cancer cells can have such a mutational burden.

CNAs are generated by various mechanisms ${ }^{6,7}$. The major causes of arm-level CNAs are defects in chromosome segregation ${ }^{6}$, whereas mechanisms involving double-strand break repair and DNA replication have been proposed for more complicated or focal CNAs ${ }^{7}$. Recent studies have shown that massive chromosome rearrangements, such as interchromosomal translocations and chromothripsis, occur in micronuclei, which are generated by chromosome mis-segregation during mitosis, through a combination of mechanisms including replication errors ${ }^{7-10}$. However, it is largely unknown whether the mechanism of recurrent, cancer type-specific CNAs is due to structural or functional relevance.

Tumour suppressor protein adenomatous polyposis coli (APC) as a component of the APC/AXIN/GSK3 $\beta$ complex ( $\beta$-catenin destruction complex) has a well-established function in the WNT/ $\beta$-catenin pathway. Specifically, it controls $\beta$-catenin levels ${ }^{11-13}$. APC is also associated with microtubule cytoskeletons and its dysfunction induces chromosome instability (CIN) (Fig. $\mathrm{S} 1)^{14-17}$. Chromosome mis-segregation because of premature mitotic exit is commonly observed 
after APC inactivation in various cell lines and mouse ES cells ${ }^{16-19}$. Nevertheless, the mechanisms underlying this CIN remain controversial ${ }^{20}$. Moreover, it remains unclear whether and how initiation of CIN by $A P C$ mutations drives tumour progression.

Mutations in the $A P C$ gene promote tumourigenesis through constitutive activation of the $\mathrm{Wnt} / \beta$-catenin signalling pathway ${ }^{21-23}$. In the conventional model, Wnt signalling is activated immediately after homozygous loss of $A P C$, which induces upregulation and nuclear accumulation of $\beta$-catenin that is required to evoke Wnt signalling and the proliferative response ${ }^{11-13}$. However, several studies have failed to confirm nuclear accumulation of $\beta$-catenin in early lesions following $A P C$ loss, despite robust staining in later lesions of human adenomas and animal models ${ }^{24-26}$, raising the possibility of unexplained mechanisms for adenoma formation independent of $\mathrm{Wnt} / \beta$-catenin signalling at early stages ${ }^{27,28}$.

Here, we determined the functional relationship between APC deficiency and APC mutant tumour-specific CNAs through comparative analyses of APC functions in cultured cells and tumours. First, we identified the critical molecular mechanisms involving Aurora A kinase (AURKA) and $\beta$-catenin, which induce CIN and growth retardation in $A P C$ mutant cells. Next, we revealed the mechanisms that overcame the adverse effects of $A P C$ mutation by forming recurrent CNAs to improve the proliferation potential. Our findings demonstrated that $A P C$ mutant cells exploited their own CIN ability to acquire compensatory chromosome alterations required to restore cellular fitness, which provide a new insight into the mechanisms for the formation of cancer type-specific CNAs. 


\section{Results}

\section{Identification of an important phenotypic feature of mitotic spindles upon APC deficiency}

Numerous studies have reported that APC cooperates with many mitosis regulators, including spindle assembly checkpoint protein Bub1/R1 and microtubule-binding protein End-binding 1 (EB1), to ensure accurate chromosome separation ${ }^{16-18,29}$ (Fig. S1). It should be noted that depletion of APC does not significantly affect spindle formation, but leads to chromosome missegregation ${ }^{18,30}$. Despite accumulating knowledge regarding the molecular interactions, most essential mitotic functions of APC contributing to tumour progression over the long term remain undetermined ${ }^{20}$.

To quantitatively and precisely determine the overall spindle phenotype affected by APC deficiency, we employed lattice light-sheet microscopy ${ }^{31,32}$ that enables whole cell, high spatiotemporal resolution 3D live cell measurement. Using this technique, we monitored microtubule growth dynamics by visualising GFP-fused End-binding 1 (EB1-GFP), a marker of newly assembled microtubule ends ${ }^{33}$ (Fig. 1a, Fig. S2, and Movie 1-3). To detect even slight differences, we integrated numerous time point images from multiple cells in silico and found that the microtubule growth probability was reduced significantly around centrosomes after APC depletion (Fig. 1b). This result indicated that the centrosomes were immature under APC deficiency.

Consistent with this finding, accumulation of $\gamma$-tubulin at centrosomes and activation of centrosomal kinases AURKA (auto-phosphorylation at T288) and polo-like kinase 1 (PLK1; phosphorylation at $\mathrm{T} 210$ ), which are central mitotic kinases that co-operatively regulate centrosome maturation $^{34,35}$, were reduced significantly in APC-depleted cells (Fig. 1c). 


\section{APC regulates AURKA activity together with AXIN1 and $\beta$-catenin}

To determine the molecular mechanism involved in centrosome regulation by APC, we screened centrosomal proteins involved in centrosome maturation for their association with APC. We found that APC bound to AURKA, PCNT (pericentrin), and ch-TOG through its N- and/or Cterminal regions (Fig. 1d and Fig. S3). Among these interactors, AURKA has been reported to act upstream of the others to regulate centrosome maturation ${ }^{34-36}$. Therefore, we further investigated APC functions in AURKA regulation.

We did not detect promotion of AURKA activation by APC in vitro. Because AURKA interacts with AXIN1, a core scaffold in the $\beta$-catenin destruction complex with APC ${ }^{11}$, and disrupts the complex to stabilise $\beta$-catenin ${ }^{37}$ (Fig. S4a), we investigated the effect of AXIN1 and $\beta$-catenin on AURKA activation. We found that the middle portion of AXIN1 promoted AURKA activation in vitro assays and in HEK293T cells, which was similar to TPX2, a wellcharacterised activator of AURKA ${ }^{38}$ (Fig. 1e, f and Fig. S4b). Strikingly, elevated $\beta$-catenin levels inhibited AXIN1-mediated AURKA activation (Fig. 1g). The N-terminal region of APC facilitated AXIN1-mediated AURKA activation significantly (Fig. 1h). Because expression of a longer APC fragment lacking only the C-terminal region was technically difficult, we used MEFs from $A p c^{1638 T}$ mice lacking the C-terminal half of $\mathrm{APC}^{39}$ and confirmed that the Cterminal region of APC activated AURKA (Fig. S1b and 4c). Taken together, APC positively regulates AURKA activity via two mechanisms: promoting AXIN1-mediated AURKA activation and $\beta$-catenin degradation, whereas APC inactivation inhibits AURKA activation indirectly through increased levels of $\beta$-catenin. 


\section{Elevated $\beta$-catenin levels block AURKA activation at centrosomes}

In tumours and cancers, $A P C$ is genetically truncated or lost ${ }^{21,22,40,41}$ (Fig. S1). To investigate cooperation of APC with AURKA under conditions similar to the disease, we used the noncancerous p53-proficient human mammary epithelial cell line MCF10A and the MCF10Aderived cell line APC883 that harbours a homozygous APC truncation (Fig. S1b). Consistent with previous reports ${ }^{16-18}$, chromosome separation errors monitored by the appearance of lagging chromosomes were increased significantly in APC883 cells (Fig. 2a), which were associated with chromosomal defects (Fig. S5a, b and Table 1). In these cells, $\beta$-catenin levels were elevated and the activated fraction of AURKA was reduced as expected (Fig. 2a and Fig. S2b). When $\beta$-catenin levels were reduced to the same levels as those in parental cells by RNA interference (Fig. S2b), AURKA activity was restored and the frequency of chromosome separation errors was reduced by $\sim 20 \%$, although the error frequency was still significantly higher than that in parental cells (Fig. 2a, Fig. S5c). Introduction of the constitutively active form of AURKA, AURKA (T288D), but not TPX2, also partially reduced chromosome separation errors (Fig. 2a, Fig. S5d, e). In addition, it was reported that ES cells from $A p c^{1638 T}$ mice displayed weak CIN phenotype, while $\beta$-catenin was not upregulated in these mice ${ }^{16,39}$ (Fig. $\mathrm{S} 1 \mathrm{~b})$. These all results indicated that stabilisation of $\beta$-catenin is the major cause of CIN by inhibiting AURKA activation even in the presence of truncated APC, but full length APC still suppressed CIN.

As an underlying mechanism for AURKA inhibition by $\beta$-catenin, we found that stabilised $\beta$-catenin perturbed the dynamics of AURKA at centrosomes. Localisation of AXIN1 and $\beta$-catenin to centrosomes and their involvement in mitotic centrosome functioning have been reported $^{42-45}$. Exogenous expression of a dominant stable form of $\beta$-catenin (MM $\beta$-catenin), in 
which the GSK phosphorylation sites that promote destruction have multiple mutations ${ }^{11}$, in parental MCF10A cells resulted in accumulation of AURKA on spindles along with MM $\beta$ catenin, while the fraction of active AURKA was decreased (Fig. 2b and Fig. S2c). Fluorescence recovery after photobleaching (FRAP) analysis to monitor the mobility of molecules revealed that turnover of GFP-AURKA at centrosomes was delayed in MM $\beta$-catenin-expressing cells compared with cells transfected with wildtype $\beta$-catenin (Fig. 2c and Fig. S2c). These results suggested that stabilised $\beta$-catenin sequesters AURKA in non-functional complexes on spindles, which led to AURKA dysregulation (model in Fig. 2d).

\section{Upregulation of AURKA in $A P C$ mutant tumours}

To evaluate our models, we investigated the state of AURKA activation in $A P C$ mutant tumours using $A p c^{\Delta 716}$ mice that have a heterozygous $A p c$ truncation mutation that leads to severe polyposis after loss of the wildtype allele ${ }^{46}$ (Fig. S1). Analysis of multiple polyps in comparison with crypts showed that AURKA activity as a ratio of phosphorylated to total AURKA staining was unexpectedly higher in many polyps than in crypts, while $\beta$-catenin staining was increased in tumour cells (Fig. 3a, b and Fig. S6a, b). Close inspection of each polyp revealed that the average value of active AURKA varied among polyps and there was intratumoural heterogeneity (Fig. 3b). The staining intensity of TPX2 also showed an increasing trend (Fig. 3b and Fig. S6c). Consistently, in human APC mutant early stage tumours, increases of AURKA activity and TPX2 were observed concomitantly with increased proliferative activity (Fig. 3c and Fig. S7a-c). This heterogeneity was considered to be induced by genetic instability. If so, AURKA-activated cells appeared because of genomic rearrangement, which gain AURKA-activating genes. 
This assumption was in accordance with the observation that the long arm of chromosome 20 (20q), carrying AURKA and TPX2 genes are located on 20q at 20q13.2 and $20 q 11$, respectively, is frequently amplified in human colorectal cancers (CRCs) ${ }^{2}$. It is also noteworthy that chromosome $13 \mathrm{q}$, the second most frequently amplified chromosome in $\mathrm{CRCs}^{2}$, contains $B O R A$ at $13 \mathrm{q} 21.33$, which encodes another AURKA-activating factor ${ }^{47}$. Because the association of $A P C$ mutations with amplification of these loci had not been investigated, we reanalysed The Cancer Genome Atlas dataset ${ }^{2}$ for $A P C$ mutations. The results demonstrated that amplifications of $20 \mathrm{q}$ and $13 \mathrm{q}$ were significantly more frequent in cancers with $A P C$ mutations than in those without them (Fig. 4a), and APC-mutant cancers had significantly higher copy numbers of these chromosome arms (Fig. 4b).

We next investigated whether AURKA-activating CNAs are initiated at the early stage of tumourigenesis using the $A p c^{\Delta 716}$ mouse. We performed high resolution array-based comparative genomic hybridisation (aCGH) analysis to detect DNA copy number alterations in individual polyps from $A p c^{\Delta 716}$ mice. In the mouse genome, Aurka/Tpx2 and Bora loci reside on chromosomes $2 \mathrm{H} 3 / 2 \mathrm{H} 1$ and $14 \mathrm{E} 2.2$, respectively (Fig. 4c). The three examined polyps all exhibited amplification of the Tpx2, locus amplification of the Aurka locus was observed in two polyps, and amplification of the Bora locus was found in two polyps (Fig. 4c). Syntenic comparison of human and mouse chromosomes showed cross-species overlaps in the amplified regions, which suggested that these $A U R K A$-related loci were selected by functional relevance rather than structural factors.

\section{Arm-level CNAs in APC mutant tumours}


Previous cancer genome analyses of various cancer types have revealed that the most prevalent CNAs are either very short, focal CNA or arm-level CNA ${ }^{1}$. Therefore, we investigated the types of CNAs in $A p c^{4716}$ mouse tumours by fluorescence in situ hybridisation (FISH) analysis for Aurka and Col5al that are located on different sides of chromosome 2 (Fig. 4c). In more than 3,000 tumour cells observed, we did not find a cluster of amplified genes as seen for ERBB2 and $c-m y c$ in human breast cancer, which occurred by intrachromosomal amplification to form focal $\mathrm{CNAs}^{48,49}$, although the distributions of Aurka and Col5al signal numbers in tumour cells significantly shifted to a higher number than those in crypts (Fig. 5a, b). These observations indicated that amplification of the Aurka locus in $A p c^{\Delta 716}$ mouse tumours was induced by armlevel CNAs that were considered to be induced by chromosome mis-segregation.

To confirm the occurrence of chromosome mis-segregations in $A p c^{\Delta 716}$ mouse tumours, we counted micronuclei positive for phosphorylated histone H2A.X $(\gamma \mathrm{H} 2 \mathrm{~A} . \mathrm{X})$ that accumulates at high levels when containing mis-segregated and damaged chromosomes ${ }^{8,50}$. In $A p c^{\Delta 716}$ mice, the incidence of $\gamma \mathrm{H} 2 \mathrm{~A}$.X-positive micronuclei was higher in the intestinal tumours than in crypts showing normal morphology (Fig. 5c, d).

\section{AURKA-activating CNAs promote cell proliferation}

Next, we investigated why AURKA-activated CNAs were selected. Direct visualisation of the cell division cycle by live imaging revealed that the doubling time of APC883 cells was about twice as long as that of parental MCF10A cells (Fig.6a, b and Movies 4-6). Strikingly, a reduction of the $\beta$-catenin level in APC883 cells, which recovered AURKA activity (Fig. 2a), dramatically restored the doubling time to the same as that of parental MCF10A cells (Fig. 6a, b). These results indicated that blockade of AURKA activation by stabilised $\beta$-catenin was the 
major cause of the delay in the cell cycle and thus additional AURKA activation was required to proliferate. Notably, expression of dominant stable MM $\beta$-catenin in MCF10A cells did not delay the doubling time (Fig. 6b). Therefore, full length APC cancelled the adverse effects of stabilised $\beta$-catenin on cell proliferation.

Furthermore, as the passage number increased, a subgroup of APC883 cells with a higher growth rate appeared spontaneously and became dominant in the culture. These cells had acquired new abnormal karyotypes consisting of a near tetraploid chromosome content including common numerical imbalances (Fig. 6b, c; Fig. S5f and Movie 5). Remarkably, chromosome 20 was the most abundant at six copies per cell. In these cells, active AURKA was restored to levels comparable to those in parental MCF10A cells because of the increase in total AURKA levels (Fig. 6d).

\section{Delayed cell cycle progression in $A P C$ mutant cells}

Our results indicated that increased levels of $\beta$-catenin suppressed cell cycle progression in $A P C$ mutant cells. However, this notion appears to be inconsistent with the conventional understanding that tumour cells exhibit uncontrolled (fast) growth. Nevertheless, it has not been determined whether abnormal control of proliferation of $A P C$ mutant cells leads to an accelerated cell cycle. Therefore, we estimated the cell cycle rate by measuring the ratio of dividing cells in the mouse intestines. As shown in Fig. 6e, the proportion of dividing cells was significantly higher in crypts than in tumours, which suggested that these cells had a slower cell cycle rate than crypt cells undergoing normally controlled proliferation. In the mouse and human tumours used in this study, increased $\beta$-catenin signals were observed throughout the cytoplasm without obvious nuclear accumulation (Fig. S6b, 7d), which indicated that these cells were in 
this state before intense activation of Wnt signalling. This observation was consistent with their slow growth rate.

In addition, in $A p c^{4716}$ mouse treated with the AURKA inhibitor alisertib or MLN8054, the abundance of cells positive for Ki67, a proliferation marker, was reduced among tumour cells, but remained unchanged in crypts (Fig. 6f, g). These results indicated that the tumour cells were more dependent on AURKA activity.

Taken together, our results demonstrated that, upon $A P C$ mutation, the induced CIN property allowed chromosome alteration, and AURKA-activating CNAs were selected to evade the adverse effects of stabilised $\beta$-catenin, which was the first selection pressure in the formation of $A P C$ mutant CRC-specific CNAs and affected the fate of tumour cells for a long period of time (Fig. 7).

\section{Discussion}

In this study, we performed comparative analyses of APC functions in cultured cells and mouse/human tumours, and identified two critical molecular mechanisms that are important to shape the cancer genome. One is the mechanism of CIN induced by APC deficiency. APC positively regulates AURKA activity in the normal state. Upon APC dysfunction, stabilised $\beta$ catenin blocks AURKA activation, which impairs centrosome maturation and causes chromosome mis-segregation. The other is the mechanism of recurrent CNA formation generated in $A P C$ mutant tumours. AURKA blockade by stabilised $\beta$-catenin in $A P C$ mutant cells causes a proliferation delay that results in the selection of compensatory CNAs that activate AURKA. 


\section{APC cooperates with AURKA in a complex with AXIN1 and $\beta$-catenin}

Starting with quantitative 3D mapping of spindle microtubule dynamics using lattice light-sheet microscopy and computational analyses, we identified AURKA as a major dysregulated factor in APC mutant cells at centrosomes. In APC-deficient cells, the activated fraction of AURKA was reduced, which resulted in chromosome mis-segregation and a delayed cell cycle. APC activates AURKA together with Axin1, whereas APC deficiency increases the levels of $\beta$-catenin and blocks AURKA activation. Thus, APC acts as a scaffold for AURKA activation with AXIN1, which resembles the $\beta$-catenin destruction machinery ${ }^{11}$. In addition, APC itself controls AURKA independently with $\beta$-catenin, because chromosome separation error was observed in $A P C$ mutant cells having normal $\beta$-catenin levels.

Notably, the phenotype caused by AURKA inhibition in APC mutant cells, which induced CIN without cell cycle arrest, is unique because AURKA inhibition by genetic deletion or an inhibitory agent commonly leads to abnormal spindle formation and CIN followed by DNA damage-induced cell cycle arrest ${ }^{35,51}$. The mechanisms of a leaky DNA damage checkpoint in APC mutant cells should be clarified in future studies.

\section{APC functions in cell proliferation}

Our results demonstrated that increased levels of $\beta$-catenin in APC mutant cells had slowed cell cycle progression. This appears to conflict with the traditional model in which activation of Wnt/ $\beta$-catenin signalling is the initiating event underlying colon adenoma formation following loss of $A P C^{52-55}$. However, our observations are in good agreement with another hypothesis in which the proliferative response of $\beta$-catenin in $A P C$ mutant cells requires additional mutations, 
such as in $K R A S^{28,56}$, at later stages, because examinations of human early adenomas, intestinal polyps from Apc mutant rat models, and intestines from apc mutant zebrafish embryos have not confirmed the presence of nuclear $\beta$-catenin ${ }^{24-26,28}$. These all observations suggest that $A P C$ loss and upregulation of $\beta$-catenin can also be considered as independent causes of tumour initiation $^{28}$.

Importantly, exogenous expression of stable mutant $\beta$-catenin in the presence of full length APC did not exert a disadvantageous effect on cellular proliferation, which suggested that APC cancels the unfavourable effects caused by increased levels of $\beta$-catenin. This is reasonable because Wnt/ $\beta$-catenin signalling in normal developmental processes must not suppress cell proliferation.

\section{CIN in tumours}

We confirmed an increase in the frequency of chromosome separation errors in mouse tumours, which could contribute to the CNA formation. When considering CNA formation in different species, it is unlikely that the CNA formation processes are the same in humans and mice, considering the differences in human and mouse chromosomal structures. This may cause differences in the tumour phenotype and carcinogenicity as well as the efficacy of anti-cancer drugs in humans and mice. Because CIN is a major hallmark of cancer ${ }^{57,58}$, species dependence of the chromosome structure should be considered in cancer research and drug development.

\section{CNAs to restore tumour cell fitness}


Although numerical and structural alterations of chromosomes have been believed to promote tumour progression, paradoxically they are also known to decrease cellular fitness and interfere with the proliferation of untransformed cells ${ }^{4,5,59}$. It has remained uncertain why cancer cells can have such a heavy mutational burden. Our results demonstrated that $A P C$ mutations decreased cellular fitness because of adverse effects caused by stabilised $\beta$-catenin blocking AURKA activation. APC mutant cells need to increase AURKA activity to restore fitness rather than to gain additional growth advantages, which could be a major selection pressure to achieve optimal fitness through the formation of compensatory CNAs.

This kind of view of compensatory mutation has been extensively studied in the field of molecular and microbial evolutions ${ }^{60-62}$. Here, the deleterious effects of one mutation that causes fitness loss is effectively compensated by its epistatic interaction with a second mutation. Interestingly, in the bacteriophage, the probability of compensatory mutations increased with the severity of the deleterious fitness effect, while compensatory mutations have deleterious effects in a wildtype background ${ }^{63}$. In human tumours, if a considerable number of chromosome alteration events are driven out of necessity to compensate, this hypothesis may facilitate elucidation of the mechanism of recurrent CNA formation.

Collectively, in this study, we provide an example of compensatory CNAs by identifying a critical relationship between prior mutations (APC mutations) and particular CNAs (AURKAactivating CNAs such as human chromosome 20 amplification). This idea can be applied to other cancer types to unravel the precise sequence of events and underlying mechanisms of the formation of cancer type-specific CNAs. Thus, compensatory CNAs that adjust tumour cell fitness represent an important, but previously unappreciated, mechanism that influences the course of cancer genome evolution. 


\section{References}

1. Beroukhim, R. et al. The landscape of somatic copy-number alteration across human cancers. Nature 463, 899-905 (2010).

2. Cancer Genome Atlas, N. Comprehensive molecular characterization of human colon and rectal cancer. Nature 487, 330-337 (2012).

3. Myllykangas, S., Bohling, T. \& Knuutila, S. Specificity, selection and significance of gene amplifications in cancer. Semin Cancer Biol 17, 42-55 (2007).

4. Ben-David, U. \& Amon, A. Context is everything: aneuploidy in cancer. Nature reviews. Genetics 21, 44-62 (2020).

5. Holland, A.J. \& Cleveland, D.W. Losing balance: the origin and impact of aneuploidy in cancer. EMBO reports 13, 501-514 (2012).

6. Holland, A.J. \& Cleveland, D.W. Boveri revisited: chromosomal instability, aneuploidy and tumorigenesis. Nature reviews. Molecular cell biology 10, 478-487 (2009).

7. Burrell, R.A., McGranahan, N., Bartek, J. \& Swanton, C. The causes and consequences of genetic heterogeneity in cancer evolution. Nature 501, 338-345 (2013).

8. Zhang, C.Z. et al. Chromothripsis from DNA damage in micronuclei. Nature 522, 179-184 (2015).

9. Leibowitz, M.L., Zhang, C.Z. \& Pellman, D. Chromothripsis: A New Mechanism for Rapid Karyotype Evolution. Annual review of genetics 49, 183-211 (2015).

10. Janssen, A., van der Burg, M., Szuhai, K., Kops, G.J. \& Medema, R.H. Chromosome segregation errors as a cause of DNA damage and structural chromosome aberrations. Science 333, 1895-1898 (2011).

11. Stamos, J.L. \& Weis, W.I. The beta-catenin destruction complex. Cold Spring Harbor perspectives in biology 5, a007898 (2013).

12. Clevers, H. \& Nusse, R. Wnt/beta-catenin signaling and disease. Cell 149, 1192-1205 (2012).

13. Ikeda, S. et al. Axin, a negative regulator of the Wnt signaling pathway, forms a complex with GSK-3beta and beta-catenin and promotes GSK-3beta-dependent phosphorylation of beta-catenin. The EMBO journal 17, 1371-1384 (1998). 
14. Näthke, I.S., Adams, C.L., Polakis, P., Sellin, J.H. \& Nelson, W.J. The adenomatous polyposis coli tumor suppressor protein localizes to plasma membrane sites involved in active cell migration. The Journal of cell biology 134, 165-179 (1996).

15. Mimori-Kiyosue, Y., Shiina, N. \& Tsukita, S. Adenomatous polyposis coli (APC) protein moves along microtubules and concentrates at their growing ends in epithelial cells. The Journal of cell biology 148, 505-518 (2000).

16. Fodde, R. et al. Mutations in the APC tumour suppressor gene cause chromosomal instability. Nature cell biology 3, 433-438 (2001).

17. Kaplan, K.B. et al. A role for the Adenomatous Polyposis Coli protein in chromosome segregation. Nature cell biology 3, 429-432 (2001).

18. Draviam, V.M., Shapiro, I., Aldridge, B. \& Sorger, P.K. Misorientation and reduced stretching of aligned sister kinetochores promote chromosome missegregation in EB1- or APC-depleted cells. The EMBO journal 25, 2814-2827 (2006).

19. Tighe, A., Johnson, V.L. \& Taylor, S.S. Truncating APC mutations have dominant effects on proliferation, spindle checkpoint control, survival and chromosome stability. J Cell Sci 117, 6339-6353 (2004).

20. Rusan, N.M. \& Peifer, M. Original CIN: reviewing roles for APC in chromosome instability. The Journal of cell biology 181, 719-726 (2008).

21. Nishisho, I. et al. Mutations of chromosome 5q21 genes in FAP and colorectal cancer patients. Science 253, 665-669 (1991).

22. Kinzler, K.W. et al. Identification of a gene located at chromosome $5 q 21$ that is mutated in colorectal cancers. Science 251, 1366-1370 (1991).

23. Kinzler, K.W. \& Vogelstein, B. Lessons from hereditary colorectal cancer. Cell 87, 159-170 (1996).

24. Anderson, C.B., Neufeld, K.L. \& White, R.L. Subcellular distribution of Wnt pathway proteins in normal and neoplastic colon. Proceedings of the National Academy of Sciences of the United States of America 99, 8683-8688 (2002).

25. Bläker, H., Scholten, M., Sutter, C., Otto, H.F. \& Penzel, R. Somatic mutations in familial adenomatous polyps. Nuclear translocation of beta-catenin requires more than biallelic APC inactivation. American journal of clinical pathology 120, 418-423 (2003).

26. Amos-Landgraf, J.M. et al. A target-selected Apc-mutant rat kindred enhances the modeling of familial human colon cancer. Proceedings of the National Academy of Sciences of the United States of America 104, 4036-4041 (2007). 
27. Fodde, R. \& Brabletz, T. Wnt/beta-catenin signaling in cancer stemness and malignant behavior. Current opinion in cell biology 19, 150-158 (2007).

28. Phelps, R.A. et al. A two-step model for colon adenoma initiation and progression caused by APC loss. Cell 137, 623-634 (2009).

29. Banks, J.D. \& Heald, R. Adenomatous polyposis coli associates with the microtubuledestabilizing protein XMCAK. Current biology : CB 14, 2033-2038 (2004).

30. Dikovskaya, D. et al. Loss of APC induces polyploidy as a result of a combination of defects in mitosis and apoptosis. Journal of Cell Biology 176, 183-195 (2007).

31. Chen, B.C. et al. Lattice light-sheet microscopy: imaging molecules to embryos at high spatiotemporal resolution. Science 346, 1257998 (2014).

32. Yamashita, N. et al. Three-dimensional tracking of plus-tips by lattice light-sheet microscopy permits the quantification of microtubule growth trajectories within the mitotic apparatus. Journal of biomedical optics 20, 101206 (2015).

33. Mimori-Kiyosue, Y., Shiina, N. \& Tsukita, S. The dynamic behavior of the APC-binding protein EB1 on the distal ends of microtubules. Current biology : CB 10, 865-868 (2000).

34. Marumoto, T., Zhang, D. \& Saya, H. Aurora-A - a guardian of poles. Nature Reviews Cancer 5, 42-50 (2005).

35. Lens, S.M.A., Voest, E.E. \& Medema, R.H. Shared and separate functions of polo-like kinases and aurora kinases in cancer. Nature Reviews Cancer 10, 825-841 (2010).

36. Nikonova, A.S., Astsaturov, I., Serebriiskii, I.G., Dunbrack, R.L., Jr. \& Golemis, E.A. Aurora A kinase (AURKA) in normal and pathological cell division. Cellular and molecular life sciences : CMLS 70, 661-687 (2013).

37. Xia, Z. et al. AURKA governs self-renewal capacity in glioma-initiating cells via stabilization/activation of beta-catenin/Wnt signaling. Molecular cancer research : MCR 11, 1101-1111 (2013).

38. Bayliss, R., Sardon, T., Vernos, I. \& Conti, E. Structural basis of Aurora-A activation by TPX2 at the mitotic spindle. Mol Cell 12, 851-862 (2003).

39. Smits, R. et al. Apc1638T: a mouse model delineating critical domains of the adenomatous polyposis coli protein involved in tumorigenesis and development. Genes \& development 13, 1309-1321 (1999).

40. Fodde, R. \& Smits, R. Disease model: familial adenomatous polyposis. Trends in molecular medicine 7, 369-373 (2001). 
41. Fearnhead, N.S., Britton, M.P. \& Bodmer, W.F. The ABC of APC. Human molecular genetics 10, 721-733 (2001).

42. Fumoto, K., Kadono, M., Izumi, N. \& Kikuchi, A. Axin localizes to the centrosome and is involved in microtubule nucleation. EMBO reports 10, 606-613 (2009).

43. Kim, S.M. et al. Axin localizes to mitotic spindles and centrosomes in mitotic cells. Experimental cell research 315, 943-954 (2009).

44. Ruan, K. et al. PLK1 Interacts and Phosphorylates Axin That Is Essential for Proper Centrosome Formation. PloS one 7 (2012).

45. Kaplan, D.D., Meigs, T.E., Kelly, P. \& Casey, P.J. Identification of a role for beta-catenin in the establishment of a bipolar mitotic spindle. Journal of Biological Chemistry 279, 1082910832 (2004).

46. Oshima, M. et al. Loss of Apc heterozygosity and abnormal tissue building in nascent intestinal polyps in mice carrying a truncated Apc gene. Proceedings of the National Academy of Sciences of the United States of America 92, 4482-4486 (1995).

47. Hutterer, A. et al. Mitotic activation of the kinase Aurora-A requires its binding partner Bora. Developmental cell 11, 147-157 (2006).

48. Blancato, J., Singh, B., Liu, A., Liao, D.J. \& Dickson, R.B. Correlation of amplification and overexpression of the c-myc oncogene in high-grade breast cancer: FISH, in situ hybridisation and immunohistochemical analyses. British journal of cancer 90, 1612-1619 (2004).

49. Kallioniemi, O.P. et al. ERBB2 amplification in breast cancer analyzed by fluorescence in situ hybridization. Proceedings of the National Academy of Sciences of the United States of America 89, 5321-5325 (1992).

50. Crasta, K. et al. DNA breaks and chromosome pulverization from errors in mitosis. Nature 482, 53-58 (2012).

51. Sasai, K. et al. Targeted disruption of Aurora A causes abnormal mitotic spindle assembly, chromosome misalignment and embryonic lethality. Oncogene 27, 4122-4127 (2008).

52. Bienz, M. \& Clevers, H. Linking colorectal cancer to Wnt signaling. Cell 103, 311-320 (2000).

53. Clevers, H. Wnt/beta-catenin signaling in development and disease. Cell 127, 469-480 (2006). 
54. Romagnolo, B. et al. Intestinal dysplasia and adenoma in transgenic mice after overexpression of an activated beta-catenin. Cancer research 59, 3875-3879 (1999).

55. Harada, N. et al. Intestinal polyposis in mice with a dominant stable mutation of the betacatenin gene. The EMBO journal 18, 5931-5942 (1999).

56. Janssen, K.P. et al. APC and oncogenic KRAS are synergistic in enhancing Wnt signaling in intestinal tumor formation and progression. Gastroenterology 131, 1096-1109 (2006).

57. Negrini, S., Gorgoulis, V.G. \& Halazonetis, T.D. Genomic instability--an evolving hallmark of cancer. Nature reviews. Molecular cell biology 11, 220-228 (2010).

58. Michor, F., Iwasa, Y., Vogelstein, B., Lengauer, C. \& Nowak, M.A. Can chromosomal instability initiate tumorigenesis? Semin Cancer Biol 15, 43-49 (2005).

59. Williams, B.R. et al. Aneuploidy affects proliferation and spontaneous immortalization in mammalian cells. Science 322, 703-709 (2008).

60. Kimura, M. The role of compensatory neutral mutations in molecular evolution. J. Genet. 64, 7-19 (1985).

61. Pal, C., Papp, B. \& Lercher, M.J. An integrated view of protein evolution. Nature reviews. Genetics 7, 337-348 (2006).

62. DePristo, M.A., Weinreich, D.M. \& Hartl, D.L. Missense meanderings in sequence space: a biophysical view of protein evolution. Nature reviews. Genetics 6, 678-687 (2005).

63. Poon, A. \& Chao, L. The rate of compensatory mutation in the DNA bacteriophage phiX174. Genetics 170, 989-999 (2005). 
Figure 1 Kawasaki et al.

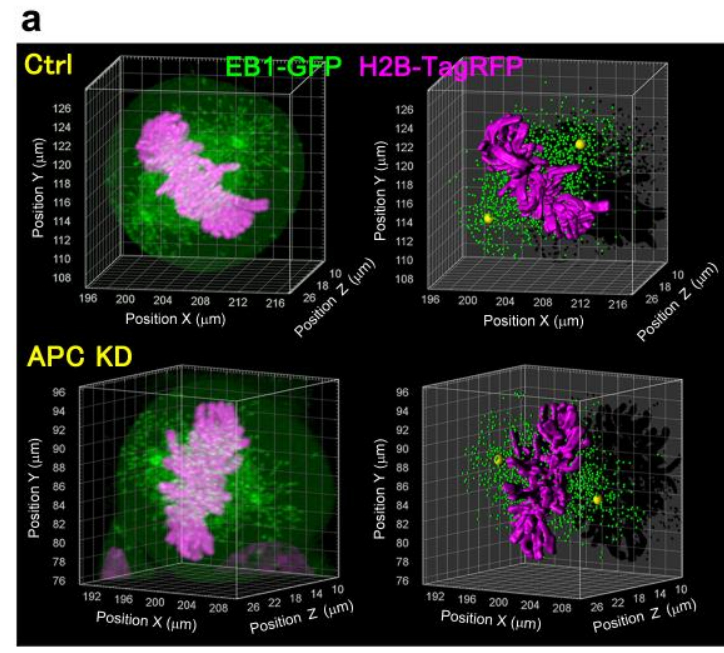

C
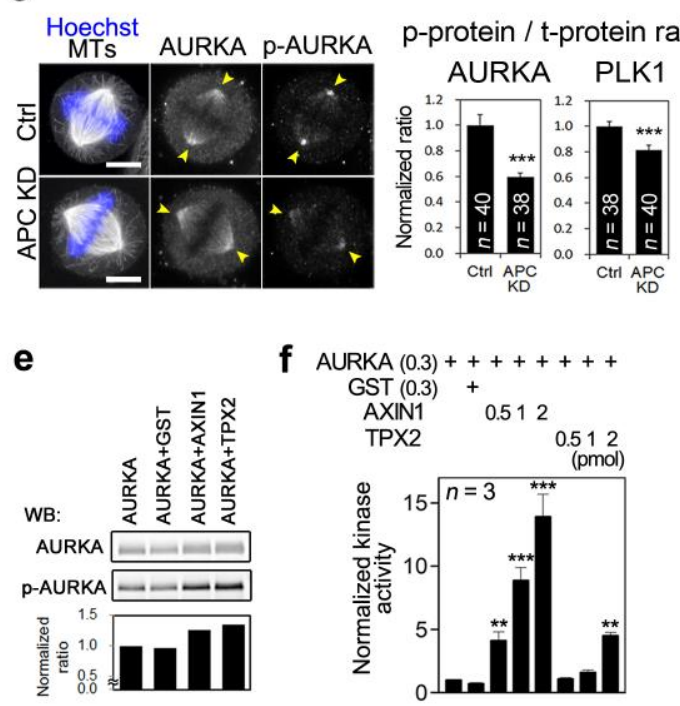

f AURKA $(0.3)++++++++$

$$
\begin{aligned}
& \text { GST ( }(0.3)+ \\
& \text { AXIN1 } \\
& \text { TPX2 }
\end{aligned}
$$

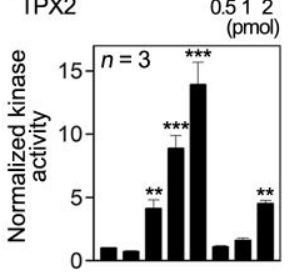

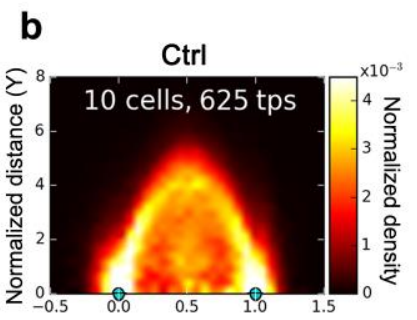

APC KD

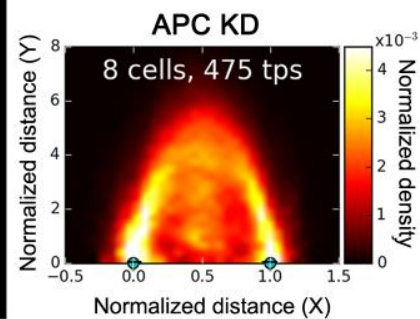

d
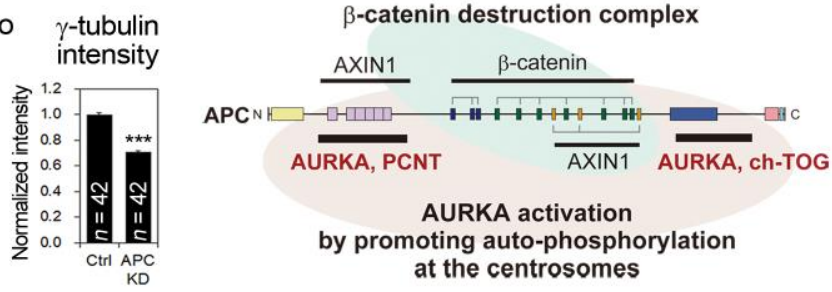

by promoting auto-phosphorylation at the centrosomes

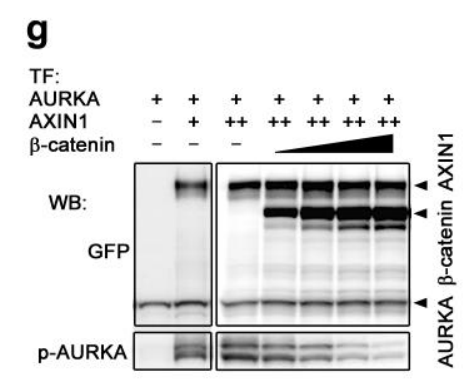

Fig. 1 Identification of the site affected by APC depletion and regulation of AURKA by

APC. (a) Three-dimensional representation of control (Ctrl) and APC-depleted (APC KD)

mitotic HeLa cells expressing EB1-GFP and H2B-TagRFP as visualized by lattice light sheet microscopy. Original images (left) and examples of automated detection of EB1-GFP positions and chromosome surfaces (right) are shown. See also Movies 1-3. (b) Rotationally projected density map (left) and density difference map (lower right). The projection method is shown diagrammatically in the upper right panel. The centrosome positions are indicated by green dots 
at positions 0.0 and 1.0 on the $\mathrm{x}$-axis. The number of cells and time points (tps) in time lapse videos used are indicated in the panel. (c) Immunostaining of AURKA and p-T288 AURKA (left). Ratios of fluorescence intensities (phosphorylated vs total protein) for AURKA (T288) and PLK1 (T210), and fluorescence intensities of $\gamma$-tubulin at centrosomes (right) are shown. Scale bars: $5 \mu \mathrm{m}$. (d) Binding sites for centrosomal proteins and components of the $\beta$-catenin destruction complex. (e) In vitro AURKA auto-phosphorylation at T288 detected by western blotting. (f) In vitro kinase assay of AURKA auto-phosphorylation. (g, h) HEK293T cells were transfected with GFP-fused AURKA, AXIN1, $\beta$-catenin, and APC fragments, as indicated, and then subjected to western blotting using the indicated antibodies. Data are shown as means $\pm \mathrm{SE}$; $* * \mathrm{P}<0.01 ; * * * \mathrm{P}<0.001$, Student's t-test. 
Figure 2 Kawasaki et al.

a

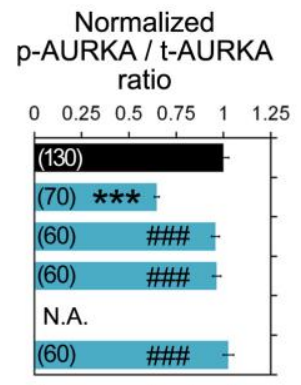

( $n$, sample size)

* vs APC-full ; \# vs APC883

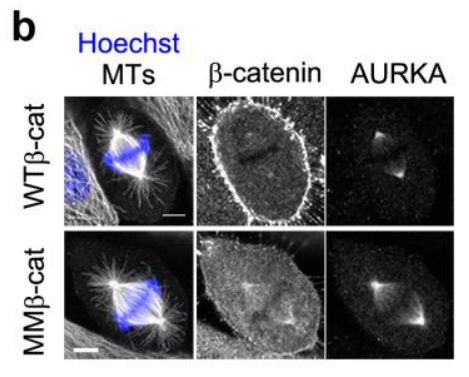

Chromosome separation (\%)

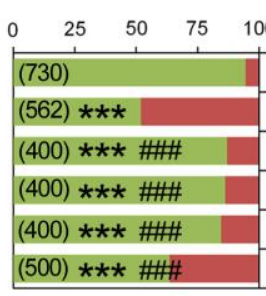

Normal

- Lagging chromosome

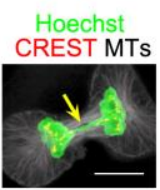

MCF10A

APC883 + $\beta$-catenin shRNA\#1 APC883 + $\beta$-catenin shRNA\#2 APC883 + AURKA(T288D) APC883 + TPX2
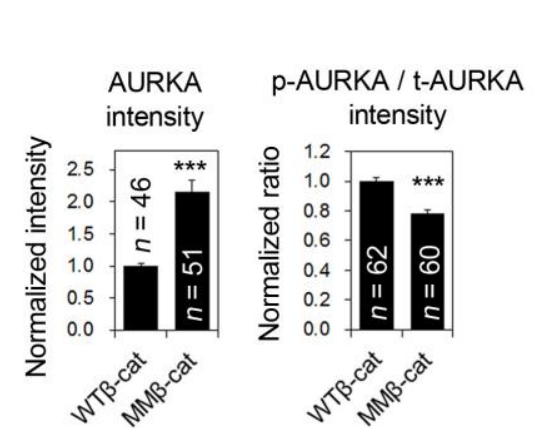

C

GFP-AURKA FRAP analysis
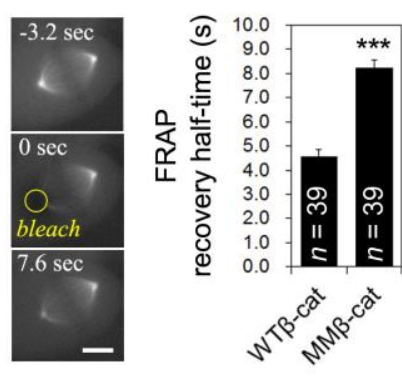

d

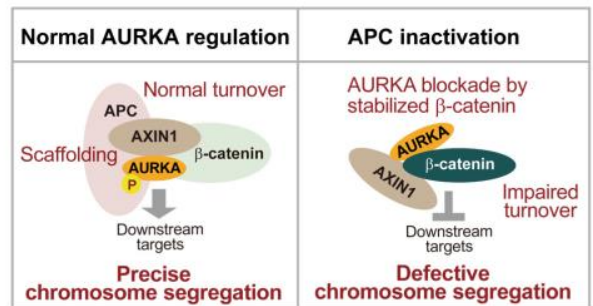

Fig.2 Stabilized $\beta$-catenin blocks AURKA activation. (a) AURKA auto-phosphorylation ratio and mitotic error rate in parental MCF10A cells expressing full length APC, APC883 cells, and cell lines derived from APC883 cells as indicated. Mitotic errors were monitored by generation of ragging chromosomes (upper right). (b) Representative images of immunostained MCF10A cells expressing myc-WT $\beta$-catenin or myc-MM $\beta$-catenin (left) and signal intensity analysis (right). (c) FRAP analysis of GFP-AURKA in cells expressing WT $\beta$-catenin or MM $\beta$-catenin. Representative images (left) and average FRAP recovery half-time (right) are shown. (d) Schematic diagram showing AURKA regulation by the APC/AXIN1/ $\beta$-catenin complex. Scale bars: $5 \mu \mathrm{m}$. Data are shown as means \pm SE. For $(\mathrm{a}),{ }^{* * *} \mathrm{P}<0.001$ vs MCF10A, ${ }^{\# \#} \mathrm{P}<0.001$ vs APC883, Student's t-test. For others, ${ }^{* * * P}<0.001$, Student's t-test. 
Figure 3 Kawasaki et al.

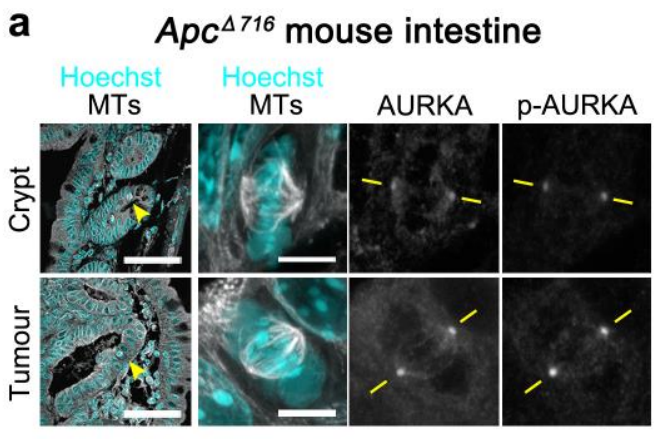

\section{Human low-grade adenoma}

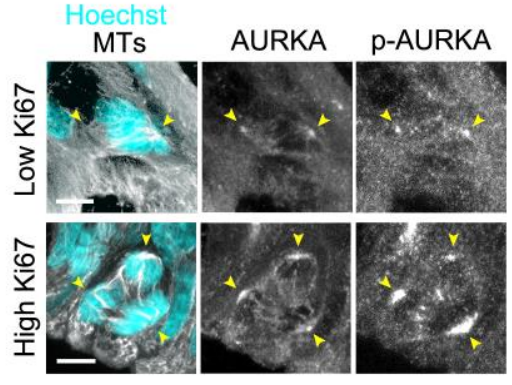

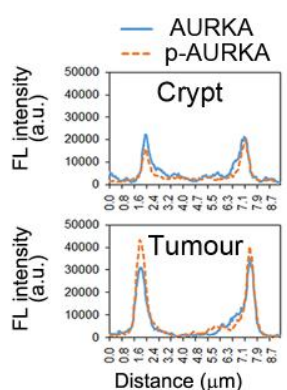

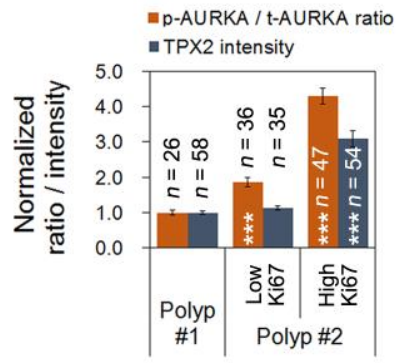

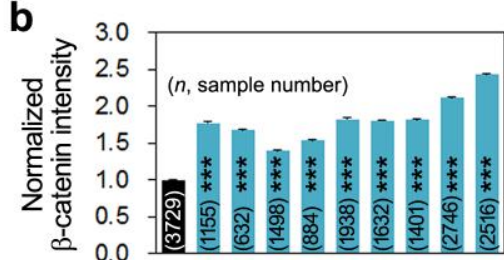
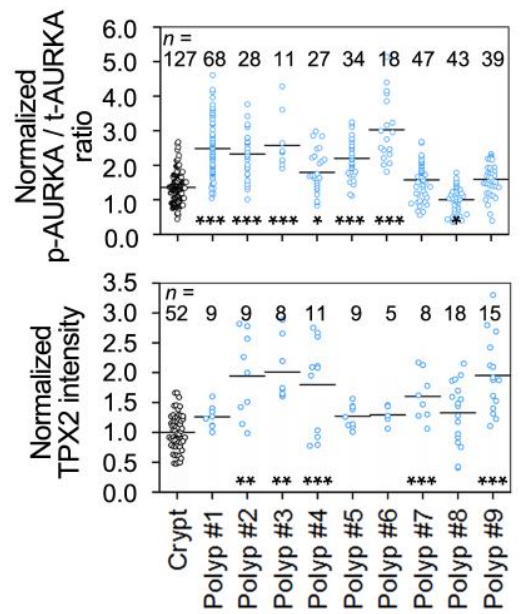

Fig. 3 AURKA activation in mouse/human tumours. (a) Representative images of mitotic cells in $A p c^{4716}$ mouse intestines stained for AURKA and p-AURKA (left), and line profiles of fluorescence intensities along the centrosomal axis (right). (b) Quantitative analysis of immunofluorescence signals in crypts and tumour tissues of nine polyps. See also Fig. S6. (c) Representative images of mitotic cells in human polyp \#2 (left). See also Fig. S7. Comparison of the phospho-to-total ratio of AURKA and TPX2 signals in polyp \#1 without APC mutation, and two regions in polyp \#2 (right). Scale bars: $50 \mu \mathrm{m}$ (a, left), $5 \mu \mathrm{m}$ (a, middle; c). Data are shown as means $\pm \mathrm{SE} ;{ }^{*} \mathrm{P}<0.05 ;{ }^{* *} \mathrm{P}<0.01 ;{ }^{* * *} \mathrm{P}<0.001$; Student's t-test (b, top; c); Tukey-Kramer test ( $\mathrm{b}$, middle and bottom). 
Figure 4 Kawasaki et al.

\section{a TCGA COAD / READ total case number: 402}

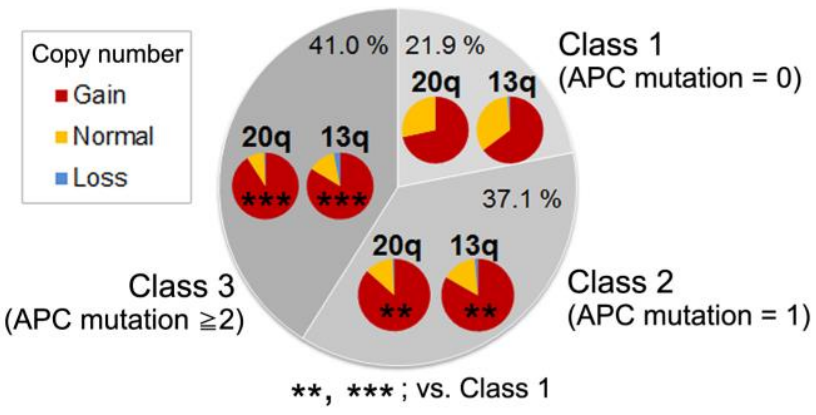

b

Chromosome 20q Chromosome 13q
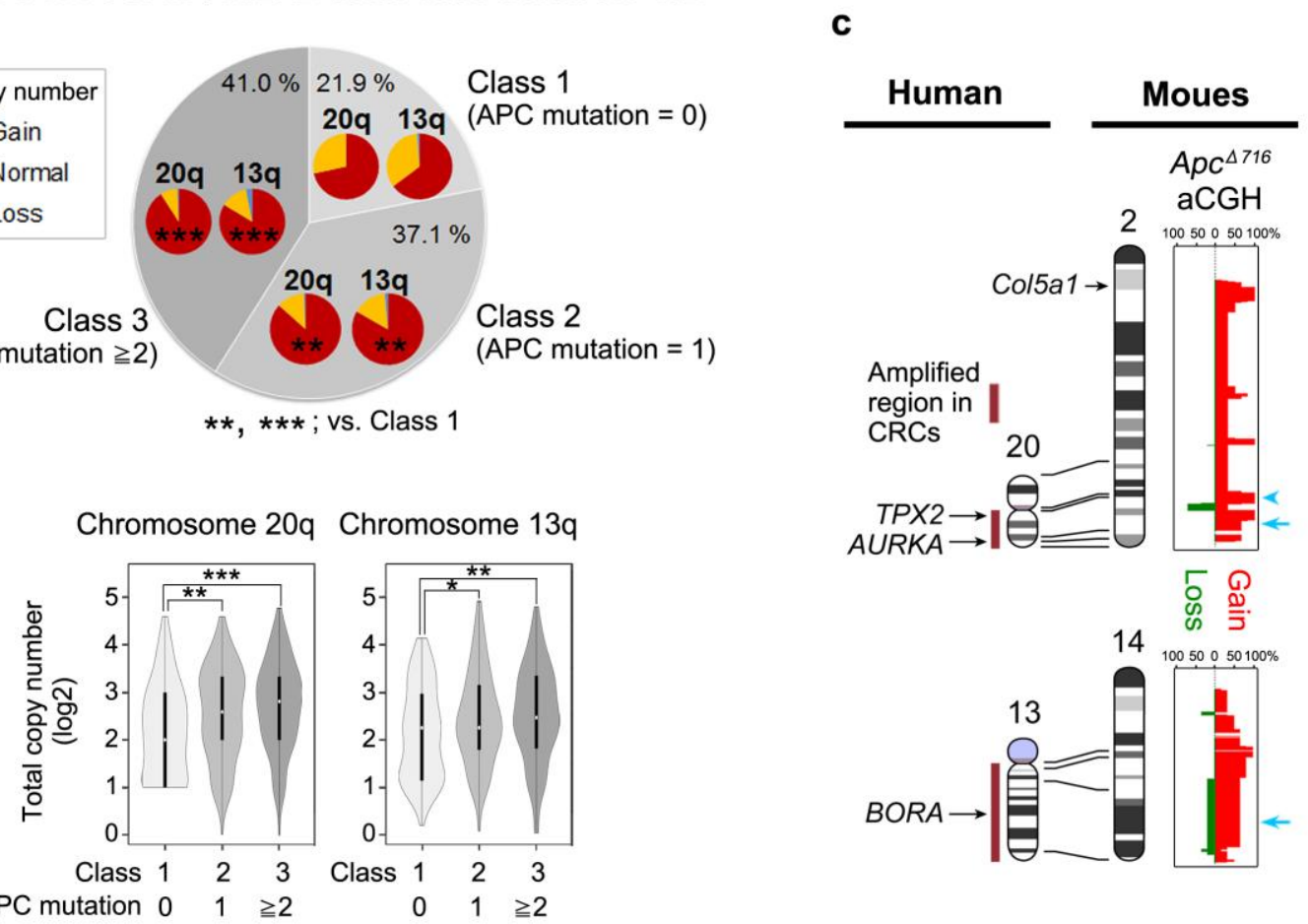

Fig. 4 Recurrent CNAs in APC mutant human CRCs and mouse tumours. (a) Correlation between the $A P C$ mutation number and copy number alteration of chromosomes $20 \mathrm{q}$ and $13 \mathrm{q}$ in human CRCs, obtained by re-analysis of the TCGA dataset. (b) Distribution of total copy numbers of chromosomes $20 \mathrm{q}$ and $13 \mathrm{q}$ in the three classes as shown by a violin plot. (c) Synteny alignment of human chromosomes 20 and 13 and mouse chromosomes 2 and 14, and aCGH analysis of $A p c^{4716}$ mouse polyps shown as frequency plots. Red and green indicate regions of gain and loss, respectively. Positions of mouse Tpx2 (arrowhead), Aurka (arrow, top), Bora (arrow, bottom) are shown. 


\section{Figure 5 Kawasaki et al.}

a

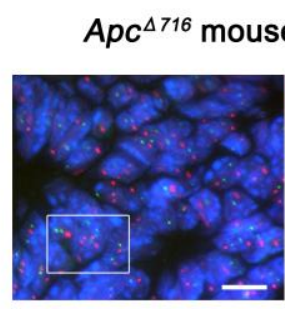

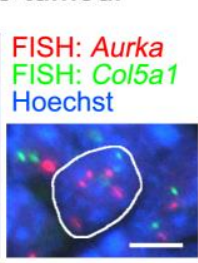
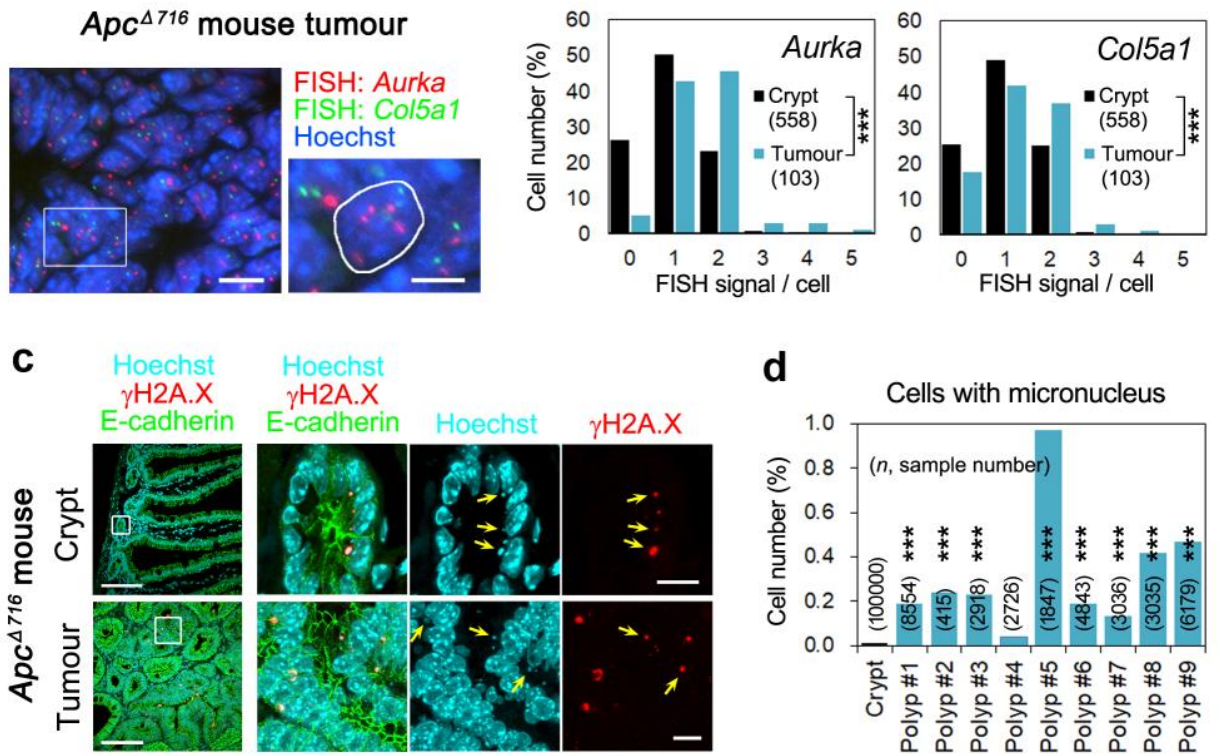

Hoechst
$\gamma$ H2A.X

E-cadherin Hoechst

$\gamma \mathrm{H} 2 \mathrm{~A} \cdot \mathrm{X}$
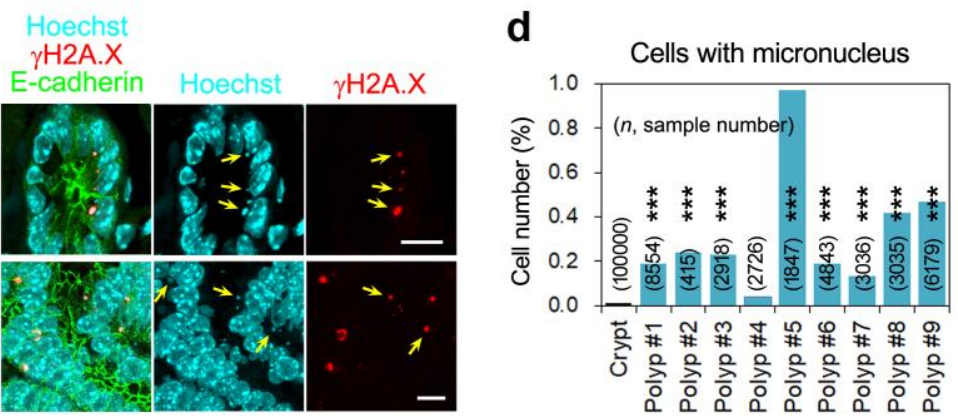

Fig. 5 Chromosome number imbalance and mitotic errors in the intestinal tumours. (a)

Representative image of FISH analysis for Aurka (red) and Col5al (green) in an $A p c^{4716}$ mouse intestinal tumour. See also Fig. 4c. The boxed area in the left is enlarged in the right. A nucleus with five Aurka spots and three Col5al spots is encircled. (b) Distribution of the FISH signal number in each cell of a tumour and crypts. The sample number is shown in parentheses. (c) Representative images of micronuclei positive for $\gamma \mathrm{H} 2 \mathrm{~A}$.X in a crypt and tumour from an $A p c^{4716}$ mouse (arrows at the left). (d) Percentages of cells with $\gamma \mathrm{H} 2 \mathrm{~A}$.X-positive micronuclei in crypts and tumour tissues in each polyp. Scale bars: $100 \mu \mathrm{m}$ (c, left), $10 \mu \mathrm{m}$ (a, left; c, right), and $5 \mu \mathrm{m}$ (a, right). $* * * \mathrm{P}<0.001 ;$ F-test (b); Student's t-test (d). 
Figure 6 Kawasaki et al.

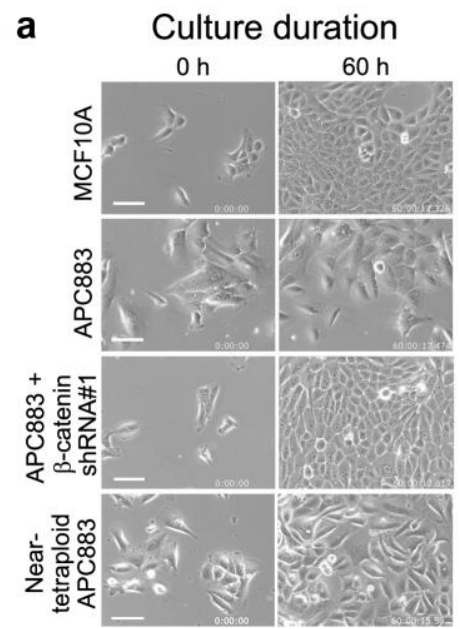

b
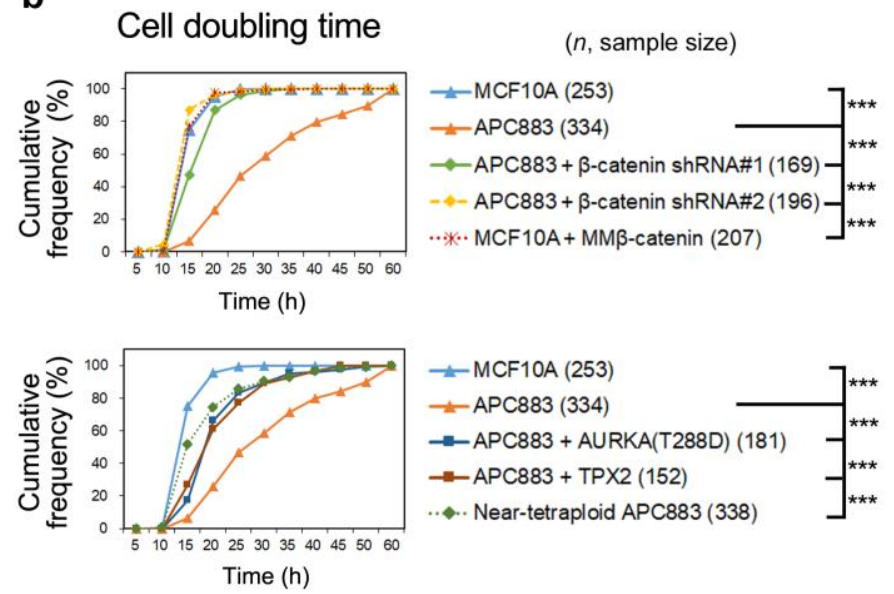

C Near-tetraploid APC883 karyotype

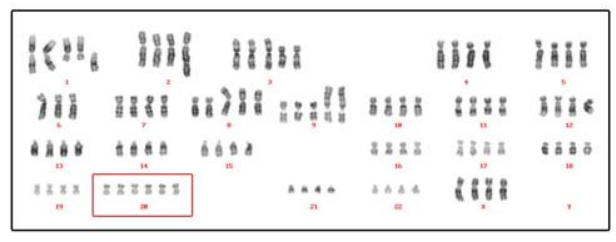

d
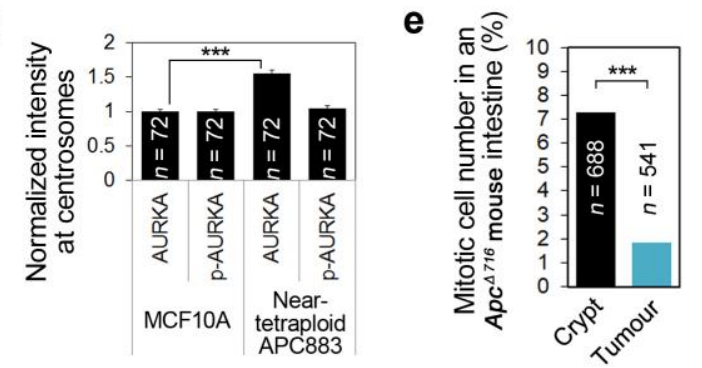

f $\quad A p c^{\Delta 716}$ mouse intestine
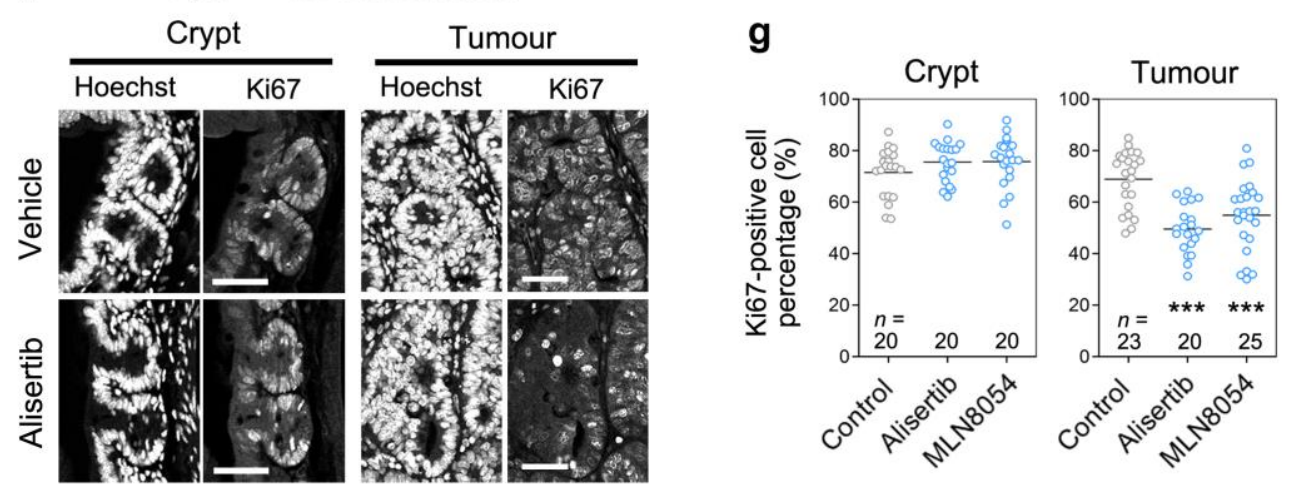

Fig. 6 Requirement of AURKA activity for proliferation of $\boldsymbol{A P C}$-mutant cells. (a) Selected images from phase contrast time-lapse videos of the indicated cell lines. The first frames $(0 \mathrm{~h})$ and frames at 60 hours later $(60 \mathrm{~h})$ are shown. See also Movies 4-6. (b) Duration of the doubling time of each cell line as shown in a cumulative histogram. (c) Representative images of the karyotype of a spontaneously appeared near-tetraploid APC883 cell derivative containing six 
copies of chromosome 20 (red box). See also Fig. S5. (d) AURKA auto-phosphorylation ratio in parental MCF10A cells and near-tetraploid APC883 cells. (e) Population of mitotic cells in $A p c^{\Delta 716}$ mouse intestines, used to estimate the cell cycle rate. (f) Representative images of Ki67labeled tissue sections of $A p c^{\Delta 716}$ mice treated with vehicle or alisertib. (g) Ki67-positive cell population in $A p c^{\Delta 716}$ mice treated with vehicle or AURKA inhibitors ( $20 \mathrm{mg} / \mathrm{kg}$ alisertib or 30 $\mathrm{mg} / \mathrm{kg}$ MLN8054) for 14 consecutive days. Scale bars: $20 \mu \mathrm{m}$ (a); $50 \mu \mathrm{m}$ (f). Data are shown as means \pm SE. $* * * \mathrm{P}<0.001$ vs APC883, the Tukey-Kramer method (b); ***P $<0.001$, Student's t-test $(\mathrm{d})$; chi-square test, $* * * \mathrm{P}<0.001$, the chi-square test $(\mathrm{e}) ; * * * \mathrm{P}<0.001$ the Tukey-Kramer $\operatorname{method}(\mathrm{g})$. 
Figure 7 Kawasaki et al.

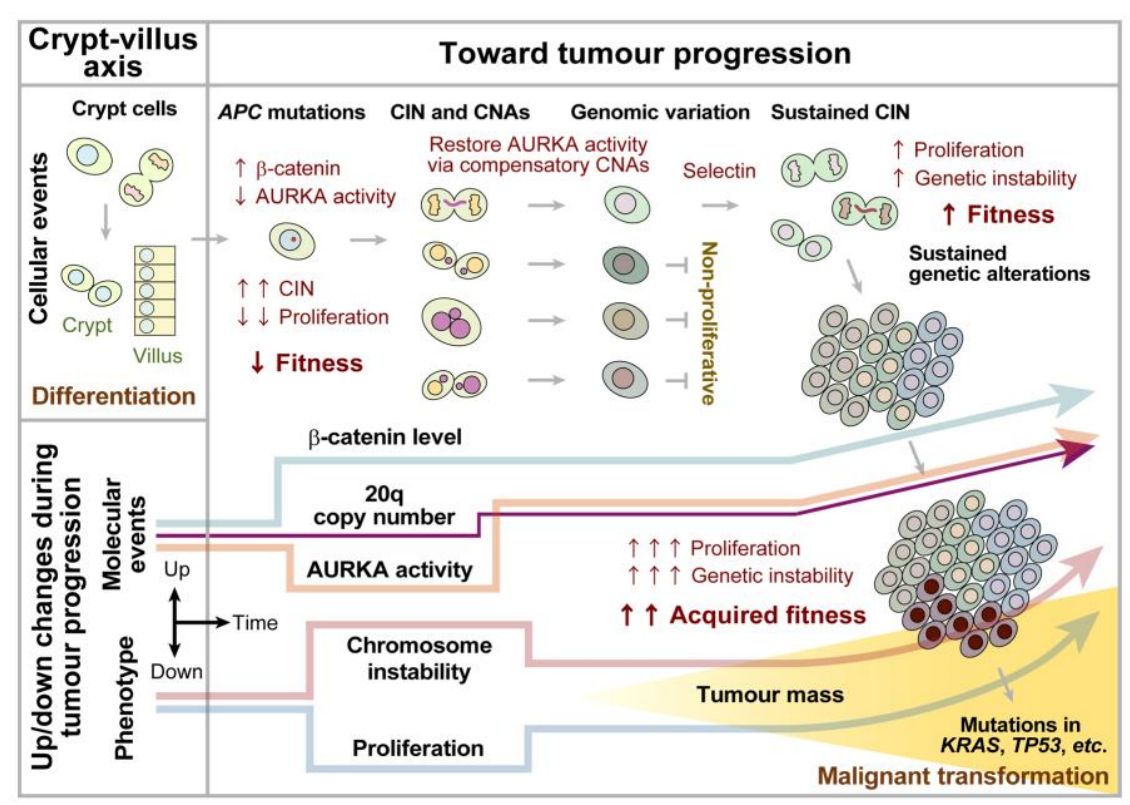

Fig. 7 Model showing the steps of malignant transformation during early tumourigenesis.

The normal crypt-villus axis and tumour progression pathway are divided, and cell phenotypes are illustrated at the top. The time course of changes in molecular activities and cell/tumour phenotypes are shown at the bottom as diagrammatic line plots. Upon APC inactivation, the CIN resulting from AURKA dysregulation generates cells carrying various chromosomal aberrations and confers a fitness penalty ${ }^{4}$. Some cells restore fitness via AURKA-activating CNAs, which may contribute to the formation of $A P C$ mutant cancer specific CNAs. 


\section{Methods}

\section{Cell culture and treatment}

All cell lines tested negative for mycoplasma contamination. Parental HeLa cell line was authenticated by Bio-Synthesis Inc (Cell Culture STR Profiling and Comparison Analysis (Cat. No.:CL1003). Other cell lines used were obtained from reliable distributors. HeLa cells and HeLa cells expressing EB1-GFP and H2B-TagRFP (clone A1) were maintained as previously described $^{64,65}$. A parental MCF10A cell line and an MCF10A cell line with the APC gene knocked out (MCF10A cells APC ${ }^{--}$) were obtained from Sigma-Aldrich-Aldrich (CLLS1069, St. Louis, MO, USA). The $\mathrm{APC}^{--}$cells, which were generated by zinc finger nuclease (ZFN) technology, are homozygous for a 17 bp deletion (GCAGCCCAGATTGCCAA) in the endogenous $A P C$ gene at 2650-2666 bp of transcript variant 3 (NM_000038). This mutation results in a truncated 883-amino acid APC protein followed by an unrelated 21 amino acid sequence at its C-terminus (SHGRSVSHSYLSGRQKFWVYH). In this paper we refer to this cell line as APC883. The HEK293-based Lenti-X 293T cell line was obtained from Takara Bio (Shiga, Japan) and was cultured in DMEM (Thermo Fisher Scientific/Gibco, Waltham, MA, USA) supplemented with $10 \% \mathrm{FCS}$ in a 5\% $\mathrm{CO}_{2}$ atmosphere. Mouse embryonic fibroblasts (MEFs) of wild-type C57BL/6 mice and homozygous $A p c^{1638 T}$ mice were isolated from embryonic day (E) 13.5 embryos, and were cultured in DMEM supplemented with 10\% FCS in a $5 \% \mathrm{CO}_{2}$ atmosphere. The number of cells was counted using an automated cell counter (Countess; Invitrogen, Carlsbad, CA, USA).

\section{Lattice light-sheet microscopy and data analysis}


3D live imaging with lattice light-sheet microscopy and quantitative data analysis were performed as described previously ${ }^{31,32}$. Briefly, time-lapse images of HeLa cells stably expressing EB1-GFP and H2B-TagRFP (clone A1) ${ }^{65}$ were collected at 0.755 s intervals for EB1GFP only single channel imaging or at $1.550 \mathrm{~s}$ intervals for EB1-GFP and H2B-TagRFP dual channel imaging in fast scanning mode $(3 \mathrm{D}$ voxel pitch: $0.100 \times 0.100 \times 0.217 \mu \mathrm{m})$, or at 10.000 s intervals for EB1-GFP and H2B-TagRFP dual channel imaging in high-resolution mode (3D voxel pitch: $0.100 \times 0.100 \times 0.195 \mu \mathrm{m})$. The former data were used for quantitative analyses and the latter data were used to visualize mitosis progression. The data were displayed in 3D and processed using Imaris software (Bitplane, Zurich, Switzerland). Supplementary Video editing of projected images exported from Imaris and image processing were performed using ImageJ software, QuickTime software (Apple. Cupertino, CA, USA) and Adobe Photoshop software (Adobe, San Jose, CA, USA).

For interpretation of multi-dimensional data, images were processed for visualization and mathematical computation. The XYZ coordinate positions of EB1-GFP comets were automatically detected and the results are presented as spots or density, or analyzed mathematically using a program developed with MATLAB (MathWorks, Natick, MA, USA) as described previously ${ }^{32}$ and Python using libraries NumPy, Math and Matplotlib. An EB1-GFP comet density map (Fig. 1b) was created from the $2 \mathrm{D}$ rotational projected comet numbers and the volume associated with the $2 \mathrm{D}$ grid. To create a $2 \mathrm{D}$ rotational projection of all comets, first, coordinates of comets and centrosomes were transformed such that the spindle axis (vector connecting the two centrosomes) aligned with the x-axis of the Cartesian coordinate. Then, all comet points were rotated around the $\mathrm{x}$-axis and projected onto an arbitrary 2D plane with its normal vector orthogonal to the spindle axis. The result is a half spindle consisting of comets and 
centrosomes in 2D coordinates. Before assembling comet points from all cells, the $\mathrm{x}$ coordinates of $2 \mathrm{D}$ comets were rescaled such that inter-centrosomal distance was one. Following that, a regular 2D grid covering all comet spots was created and the number of comets in each grid element was counted. Average comet density of each element was calculated by normalizing the number of comets with i) time-lapse duration and ii) calculation of 3D volume corresponding to each grid element. The latter is a function of distance from the spindle axis and can be expressed as $v=\pi h^{2} w(2 n-1)$ where $n=1,2,3, \ldots, N y$, with $N y$ representing the total number of grid elements perpendicular to the spindle axis. $w$ and $h$ are the width and height of the elements, respectively. For cell type comparison, comet density was normalized such that the summed density of all elements was one. Normalized density was then rescaled by the global maximum to give a value between zero and one. Rescaled normalized comet density of each element and interpolated density values of in-between grid elements were color-coded and plotted. Because the centrosomes have much higher densities relative to other regions, we imposed an upper cut-off color value to better illustrate the density variation of non-centrosomal regions. The density difference graph was obtained by taking the difference between normalized density of each grid element of APC KD and control cells.

\section{Antibodies and immunolabeling}

No untested antibody was used. Rat anti-E-cadherin mAb (ECCD-2 ${ }^{66}$ ) was a kind gift from Dr. M. Takeichi (RIKEN CDB). The following commercial primary antibodies were used: mouse anti-APC (Ab-1) mAb for western blotting, clone FE9 (OP44; Lot\# D00138185; EMD Millipore/Calbiochem, Billerica, MA, USA); mouse anti-Aurora A mAb, clone 35C1 (ab13824; Lot\# GR121501-2; Abcam, Cambridge, UK); rabbit anti-Aurora A (phospho T288) polyclonal antibody (pAb), (ab195748, Lot\# GR212301-1; Abcam); rabbit phospho-Aurora A 
(Thr288)/Aurora B (Thr232)/Aurora C (Thr198) (D13A11) mAb (\#2914; Lot\# 3; CST, Danvers, MA, USA); mouse anri-PLK1 mAb, clone 36-298 (ab17057; Lot\# GR136555-1; Abcam); rabbit anti-PLK1 (phospho T210) mAb, clone EPNCIR167 (ab155095; Lot\# YJ092901CS; Abcam); rabbit anti-AXIN1 mAb, clone C76H11 (\#2087; Lot\# 4; CST); purified human anti-Centromere Protein IgG (CREST) (15-235; Lot\# 441.13BK.20; Antibodies Incorporated, Davis, CA, USA); rabbit anti- $\beta$-catenin pAb (C2206; Lot\# 059K4754; Sigma-Aldrich); mouse anti- $\beta$-catenin mAb, clone 14 (610154; Lot\# 5113978; BD Transduction Lab.); rabbit anti-Ki67 pAb (ab15580; Lot\# GR264768-1; abcam); rabbit anti-TPX2 pAb (HPA005487; Lot\# A104169; Sigma); mouse antiTPX2 mAb, clone 18D5-1 (ab32795; Lot\# GR245658-1; abcam); mouse anti- $\alpha$-tubulin, clone DM1A (T9026; Lot\# 078K4763; Sigma-Aldrich); rat anti-tubulin mAb, clone YL1/2 (ab6160; Lot\# GR57687-1; Abcam); rabbit anti- $\gamma$-tubulin pAb (ab11320; Lot\# 628882; Abcam); mouse anti- $\gamma$-tubulin mAb, clone GTU-88 (T6557; Lot\# 079K4861; Sigma-Aldrich); mouse antiGAPDH antibody-loading control (HRP), clone mAbcam 9484 (ab9482; Lot\# GR149747-1; Abcam); rabbit anti-GFP, HRP-conjugated (598-7; Lot\# 002; MBL, Aichi, Japan ); mouse antiGST, clone B-14 (sc-138; Lot\# K1808; Santa Cruz Biotechnology); mouse anti-penta-His antibody (34660; Lot\# 136244018; Qiagen); mouse anti-Myc tag mAb, clone 4A6 (05-724; Lot\# DAM1724025; Millipore); mouse anti-Myc tag mAb, clone 4A6, peroxidase-conjugated (16213; Lot\# DAM1646292; Millipore); Hoechst 33342 solution (346-07951; Lot\# FN027; Dojin, Japan) was used as a nuclear marker. Immunofluorescent staining of fixed cultured cells and western blotting were performed as described previously ${ }^{64,65,67}$. Intestinal tissues from 16-weeks old $A p c^{\Delta 716}$ mice and human adenomas were fixed in formalin and processed for paraffinembedding and sectioning as described previously ${ }^{46}$. Antigen retrieval for $\beta$-catenin (610154, BD), E-cadherin (ECCD-2), AURKA, phosphor-AURKA(T288), TPX2, Ki67 and anti- $\alpha-$ 
tubulin (YL1/2) immunostaining was performed by boiling sections for $30 \mathrm{~min}$ in Tris-EDTA pH 9.0.

\section{cDNAs, plasmids and RNA interference}

The APC cDNA used was described previously ${ }^{68}$. Wild-type mouse $\beta$-catenin and dominantstable $\beta$-catenin (MM $\beta$-catenin), in which the $\mathrm{NH}_{2}$-terminal four Ser/Thr residues recognized by GSK-3 $\beta$ were substituted for Ala ${ }^{69}$ were gifts from Dr. A. Nagafuchi (Nara Medical University). A human AXIN1 cDNA was PCR cloned from MCF10A cells. cDNAs for mouse PLK1and rat AXIN1 were described previously ${ }^{13,70,71}$. The following plasmids were obtained: $p$ WZL Neo Myr Flag AURKA (addgene plasmid \# 20427), a gift from William Hahn \& Jean Zhao; mEmerald-TPX2-N-10 (addgene plasmid \#54285) and mEmerald-Beta-Catenin-20 (mouse) (addgene plasmid \#54017), gifts from Michael Davidson. AURKA(T288D) and PLK1(T210D) mutations were introduced by PCR engineering. EGFP-fused APC, $\beta$-catenin, AXIN1 and AURKA were expressed by inserting these cDNAs into pEGFP-C (Takara/Clontech). Transient transfection of cells with plasmids was performed using Effectene transfection reagent (Qiagen, Germany).

Lentiviral vectors expressing genes of interest under EF1 $\alpha$ promoters (pLVSIN-EF1aIRES-pur, pLVSIN-EF1a-IRES-bsr) were described previously ${ }^{65}$. Myc-tagged AURKA, AURKA(T288D), PLK1, PLK(T210D) and TPX2 were generated by PCR engineering and inserted into pLVSIN-EF1a-IRES series vectors. Lentiviral vectors expressing shRNAs against human $A P C$ under U6 promoters (pLVSIN-U6-pur, bsr) were described previously ${ }^{72}$. The following lentiviral plasmids were obtained: pLKO.1 puro shRNA beta-catenin (addgene plasmid \#18803, referred to in this study as $\beta$-catenin shRNA\#1), a gift from Bob Weinberg; 
pLKO.1.sh.beta-catenin.2279 (addgene plasmid \#19762, referred to in this study as $\beta$-catenin shRNA\#2), a gift from William Hahn.

\section{Lentiviral gene transfer}

Lentiviruses carrying genes of interest were generated in HEK293T(Lenti-X) cells (Takara) using Lentiviral High Titer Packaging Mix (Takara) as described previously ${ }^{65}$. Cells were infected for $24 \mathrm{~h}$ with virus in the presence of $10 \mathrm{mg} / \mathrm{ml}$ polybrene (Santa Cruz Biotechnology), washed, and allowed to recover for $48 \mathrm{~h}$ before selection with appropriate antibiotics. For selection of gene-transduced cells, Puromycin (InvivoGen, San Diego, CA, USA) and Blasticidin S (InvivoGen) were used for selection of the $b s r$ resistance gene. HeLa cells infected with APC shRNA/pLVSIN-U6-pur were maintained in 10-20 $\mu \mathrm{g} / \mathrm{ml}$ puromycin. MCF10A cells transfected with cDNA and shRNA were maintained in media containing appropriate concentrations of selection drugs: APC883 + AURKA(T288D), $0.05 \mu \mathrm{g} / \mathrm{ml}$ Blasticidin S; APC883 + $\beta$-catenin shRNA\#1, $10 \mu \mathrm{g} / \mathrm{ml}$ puromycin; APC883 + $\beta$-catenin shRNA\#2, $1 \mu \mathrm{g} / \mathrm{ml}$ puromycin; APC883 + TPX2, $0.01 \mu \mathrm{g} / \mathrm{ml}$ puromycin. To screen for genes that are able to stimulate proliferation of APC883 cells as a results of rescue of mitotic defects and delayed doubling time, APC883 cells were screened following transfection by continuous culture for more than a month without drug selection, allowing their unconstrained proliferation. For prolonged culture, the rescued cells were maintained in media containing appropriate selection drugs as described above.

\section{Proteins}

For bacterial expression, DNA fragments encoding APC proteins were inserted into pGEX-5X-1 (GE Healthcare Life Sciences, Pittsburgh, PA, USA). For in vitro translation and purification, AURKA cDNA was inserted into pcDNA3.1 (Invitrogen) and pT7-FLAG-1 expression vectors 
(Sigma-Aldrich), and protein expressed and purified with a TNT Quick Coupled

Transcription/Translation System (Promega, Madison, WI, USA) according to the

manufacturer's protocol. Purified His6- $\beta$-catenin was described previously ${ }^{73}$. Purified human

AXIN1-MYC/DDK (TP308349) and TPX2-MYC/DDK (TP305821) proteins were obtained

from OriGene Technologies (Rockville, MD, USA). GST-tagged AURORA A, Active (A28-

18G) and Inactive (A28-14G) proteins were obtained from SignalChem (Richmond, Canada).

\section{In-cell fluorescence colocalization assay, immunoprecipitation and in vitro pull- down assays}

For preliminary detection of protein-protein association, target molecules were tagged with GFP (EGFP or Emerald GFP) or RFP (TagRFP-T or mApple) and overexpressed in HEK293T cells, and their colocalization analyzed under fluorescence microscopy (in-cell colocalization assay). For in vitro pull-down assays, proteins fused to GST were synthesized in E. coli and isolated by absorption to Glutathione Sepharose (GE Health Care). GST and GST-fusion proteins (2 $\mu \mathrm{g})$ immobilized to beads were incubated for $1 \mathrm{~h}$ at $4{ }^{\circ} \mathrm{C}$ with in vitro translation products in $500 \mu$ of binding buffer (0.1\% NP-40, 50 mM HEPES (pH 7.0), $150 \mathrm{mM} \mathrm{NaCl}, 50 \mathrm{mM} \mathrm{NaF}, 5 \mathrm{mM}$ EDTA, $1 \mathrm{mM}$ DTT, $1 \mu \mathrm{g} / \mathrm{ml}$ aprotinin, $1 \mu \mathrm{g} / \mathrm{ml}$ leupeptin, $50 \mu \mathrm{g} / \mathrm{ml}$ PMSF) and then washed extensively three times with binding buffer. Proteins adhering to the beads were resolved by sodium dodecyl sulfate polyacrylamide gel electrophoresis (SDS-PAGE), transferred to a polyvinylidene difluoride membrane filters (Millipore), and analyzed by immunoblotting. For the pull-down experiments, HEK293 cells were transfected with FLAG-tagged AXIN1-DIX(600862) and extracted in lysis buffer (50 mM Tris $\mathrm{HCl} \mathrm{pH}$ 7.5, $150 \mathrm{mM} \mathrm{NaCl}, 5 \mathrm{mM}$ EDTA, $1 \%$ Triton X-100, 2 mM Na3VO4, 10 mM NaF, 1 mM DTT). Cell lysates (500 $\mu$ l) were incubated with $2 \mu \mathrm{g}$ GST or GST-APC-221-880 bound to Glutathione Sepharose beads for $1 \mathrm{~h}$ at $4^{\circ} \mathrm{C}$. The 
beads were then washed three times with wash buffer $(20 \mathrm{mM}$ Tris $\mathrm{HCl} \mathrm{pH} 8.0,150 \mathrm{mM} \mathrm{NaCl}$, 0.5\% NP-40) and boiled in SDS sample loading buffer. Protein complexes were separated by SDS-PAGE and detected by immunoblotting.

\section{In vitro kinase assay}

AURKA autophosphorylation was evaluated using the ADP-Glo Kinase Assay (Promega) according to the manufacturer's instructions. The ADP-Glo Kinase Assay is a luminescent kinase assay that measures ADP formed via a kinase reaction. Briefly, inactive AURKA (0.3 pmol; SignalChem) was mixed with various concentrations (0.5-2 pmol) of purified GST, Axin1 (OriGene Technologies) or TPX2 (SignalChem) for $10 \mathrm{~min}$ at RT. The mixture was further incubated with $50 \mu \mathrm{M}$ ATP in Reaction Buffer A (40 mM Tris, pH 7.5, $20 \mathrm{mM} \mathrm{MgCl} 2,0.1$ $\mathrm{mg} / \mathrm{ml} \mathrm{BSA}$ ) supplemented with $50 \mu \mathrm{M}$ dithiothreitol (DTT) in a $25 \mu \mathrm{l}$ volume for $30 \mathrm{~min}$ at RT. The reaction was terminated by adding $25 \mu \mathrm{l}$ of ADP-Glo Reagent. After incubation for 40 min at RT, $50 \mu \mathrm{L}$ of the Kinase Detection Reagent was added and incubated for $30 \mathrm{~min}$ at RT. The luminescence was measured using a luminometer (Berthold Technologies, Bad Wildbad, Germany). Data from three independent experiments were averaged after normalization against the control value (AURKA only) which was set to 1.0.

\section{Western blotting}

Total cell lysates were prepared in Laemmli SDS-sample buffer supplemented with Protease Inhibitor Cocktail (Nacalai Tesque, Kyoto, Japan) and protein concentrations were quantified by the DC protein assay (Bio-Rad, CA, USA). Protein concentrations were normalized if necessary and boiled for 5 min with 2-mercaptoethanol, separated by SDS-PAGE, and transferred to 0.45 $\mu \mathrm{m}$ Immobilon-P polyvinylidene fluoride membranes (Millipore). Proteins were visualized using Amersham ECL Select Western Blotting Detection Reagents (GE Healthcare) or Chemi-Lumi 
One Super reagent (Nacalai Tesque) and detected with a chemiluminescence imaging system Davinhc-Chemi (CoreBio, Taoyuan, Taiwan). For detection of phosphorylated proteins, cell lysates were immediately boiled for 5 min with 2-mercaptoethanol without concentration adjustment. The phosphorylated fractions were determined by ratiometry of phosphor/total protein intensities.

\section{Fluorescence microscopy of immunostained specimens and data analysis}

The immunostained specimens were observed using an LSM780 confocal microscope with a Plan-APOCHROMAT 63×/1.4 NA oil immersion objective, a Plan-APOCHROMAT 40×/1.3NA oil immersion objective, a Plan-APOCHROMAT 10×/0.45 NA objective, four laser lines (405 nm; Multi-Ar, 458, 488, $514 \mathrm{~nm}$; DPSS, $561 \mathrm{~nm}$; He-Ne, $633 \mathrm{~nm})$, and a highly-sensitive gallium arsenide phosphide (GaAsP) detector (Carl Zeiss) and an LSM880 confocal microscope with a Plan-APOCHROMAT 63×/1.4 NA oil immersion for SR objective, a PlanAPOCHROMAT 40×/1.3 NA oil immersion for SR objective, a Plan-APOCHROMAT 10×/0.45 NA objective, four laser lines (405 nm; Multi-Ar, 458, 488, 514 nm; DPSS, 561 nm; He-Ne, 633 $\mathrm{nm}$ ), and a GaAsP detector (Carl Zeiss). Detection and quantitative analysis of phosphorylated proteins were performed using cultured cells and paraffin-embedded tissues fixed in $4 \%$ paraformaldehyde or formalin, respectively. To compare immunostaining intensities in each cell type cultured on different cover slips, the cover slips were processed under the same conditions and intensity values were obtained as relative values against that of control cells, which was set to 1 . To compare immunostaining intensities in different tissue sections, the sections were bound to a single slide glass to be processed under the same staining conditions. For tumour cells analysis in the intestinal tissue specimens, tumour cells in each polyp were specified by their epithelial features shown with E-cadherin or $\beta$-catenin immunostaining. 
To define the percentage of cells with $\gamma \mathrm{H} 2 \mathrm{~A}$.X-positive micronuclei in mouse tumours, cells with $\gamma \mathrm{H} 2 \mathrm{~A}$.X-positive micronuclei were counted in normal crypts and each polyp throughout the entire Swiss roll section, and the total numbers were divided by the estimated total numbers of cells in total crypt in the section and in each polyp obtained from cell densities and areas, respectively. Estimated total cell numbers were 100000 for crypt, 34338 for total tumours analyzed. Sample sizes indicate the numbers of images analysed. To measure fluorescence on centrosomes, maximum intensity projections of z-stack images were produced, and centrosomal regions were manually selected to obtain total fluorescence values in the region of interest using the ImageJ software. The average fluorescence intensities and phospho-to-total protein intensities were normalized to those of controls, which were defined as 1.0. For mouse intestines, average values from crypt cells were used as standards. For $\beta$-catenin- and TPX2labeling in mouse intestine, values from crypt cells in the neighbourhood of the polyps along the intestinal tract were used as standards. For human tumours, the average values in polyp\#2 were normalized to those of polyp\#1 without APC mutation. Sample sizes indicate the numbers of cells ( $\beta$-catenin)/centrosomes (AURKA)/spindles (TPX2) analysed. To define the percentage of Ki67-positive cells in AURKA inhibitor-treated mouse tumors, paraffin-embedded tissues fixed in formalin were stained for $\beta$-catenin, Ki67, and nuclear DNA (with Hoechst 33342), imaged on a confocal microscope, and analysed using the MetaMorph software. The Ki67- and Hoechst 33342-positive areas were determined by intensity thresholding in the crypts and tumor tissues, and the percentage of Ki67-positive areas were calculated in each image obtained from multiple crypt/tumor areas. Sample sizes indicate the numbers of images analysed. To count mitotic cells in mouse intestines, the tissue sections were stained with Hoechst33342 and imaged using an 
Imager Z1 fluorescence microscope (Carl Zeiss), and total and mitotic cells were manually counted based on the shapes of chromosomes.

\section{Live imaging and data analysis}

Time-lapse phase-contrast live imaging of cells was performed using an IX81-ZDC inverted microscope with a 20× objective lens LUCPLFN 20×PH (Olympus) controlled by MetaMorph software (Molecular Devices, Sunnyvale, CA, USA). The microscope was installed in a temperature-controlled dark box and was equipped with a motorized XYZ stage, a ZDC (Z drift compensator) autofocus system, a cooled CCD camera ORCA-AG (Hamamatsu Photonics, Shizuoka, Japan), and a heat controller (Tokai Hit, Shizuoka, Japan) and gas mixer (Olympus/Tokken, Chiba, Japan) for cell culture. During imaging cells were maintained at $37^{\circ} \mathrm{C}$ in a $5 \% \mathrm{CO}_{2}$ atmosphere. Duration of mitosis and doubling time were manually measured. Timelapse images were edited using ImageJ and QuickTime Pro software (Apple). Fluorescence recovery after photobleaching (FRAP) analysis of GFP-AURKA-expressing cells was performed using a DeltaVision system and Photokinetics data analysis software (GE Healthcare, Pittsburgh, PA, USA). A centrosomal area of approximately $3 \mu \mathrm{m}$ diameter was bleached using a $488 \mathrm{~nm}$ Quantifiable Laser Module while collecting time-lapse images at $1 \mathrm{~s}$ intervals.

\section{Animals and human colorectal adenoma}

Animals were used in accordance with governmental and institutional regulations and protocols were approved by the Animal Care and Use Committee of the RIKEN Kobe Institute, Kanazawa University and the University of Tokyo. All animals were cared for in accordance with the ethical standards of the institutions. $A p c^{\Delta 716}$ mice and paraffin-embedded tissue preparation were described previously ${ }^{74}$ Apc $^{1638 T}$ mice (backcrossed to C57BL/6) were described previously ${ }^{39,75}$. 
Wild-type C57BL/6J mice were purchased from Charles River Laboratories Japan Inc.

(Kanagawa, Japan). For AURKA inhibitor treatment, wild-type and $A p c^{4716}$ mice (two mice in each group) were dosed orally twice daily for 14 consecutive days with vehicle, $20 \mathrm{mg} / \mathrm{kg}$ alisertib (S1133, Selleckchem, Houston, TX, USA), or 30 mg/kg MLN8054 (S1100, Selleckchem) as a solution in $10 \% 2-\mathrm{HP}-\beta-\mathrm{CD}^{76}$. Human colorectal polyps were excised by polypectomy at the Nico-tama Coloproctology Clinic with informed consent and the protocol was approved by the Ethical Committee of the Riken Kobe Institute. Samples were de-identified and analyzed anonymously. A small portion of the polyp was used for genomic DNA isolation using a DNeasy Blood \& Tissue Kit (Qiagen, Hilden, Germany), and the remaining portion was immediately fixed in formalin and embedded in paraffin. For genomic sequencing, the last exon of $A P C$, encoding from the armadillo repeat region to the stop codon, was cloned by PCR as a fragment of 1000 1700 bp using specific primers, inserted into pCR-Blunt II-TOPO (Thermo Fisher Scientific, Waltham, MA, USA) and analyzed by Sanger sequencing. In this study, a hyperplasia polyp without APC mutation (polyp\#1, Asian female, early 60s) and a low-grade tubular adenoma with two truncating mutations (c.2698G > T and c4558G del) (polyp\#2, Asian female, late 50s) were used. Images of hematoxylin-eosin (HE) stained sections of a formalinfixed, paraffin-embedded Swiss roll of the intestine of an $A p c^{\Delta 716}$ mouse and of human polyps were collected using a SZX12 stereomicroscope with a DF PLAPO 1.2× PF2 objective (Olympus, Tokyo, Japan) or a Primo Vert Monitor with a Plan-ACHROMAT 4×/0.10 NA objective (Carl Zeiss, Jena, Germany), respectively.

\section{Comparative genomic hybridization (CGH) microarray analysis}

CGH microarray analysis was performed by Takara Bio using a SurePrint G3 Mouse CGH Microarray Kit, $1 \times 1 \mathrm{M}$ (Agilent Technologies, CA, USA). The genomic DNAs from APC 4716 
mouse normal intestines (control) and polyps were fragmented, amplified, and then labelled with Cy3 or Cy5 dyes. Dye-swap replication experiments were performed for each polyp sample vs. control DNA. The hybridized DNAs were applied to the microarray and scanned on an Agilent SureScan Microarray Scanner. The results were analysed using the Agilent Genomic Workbench 7.0 software (Agilent Technologies); experimental parameters were set with consideration for the mosaicism of mutations in the tumour cells and the presence of a considerable amount of normal cells in each sample. For pre-processing: Feature Filter, ON; Design Filter, v2; GC correction,

ON; Centralization, ON; Intra array, ON. For Aberration Filter: $\log _{2}$ Ratio $\geqq 0.01$; minimum

number of probes in region, 5. For analysis, the AMD-2 algorithm with threshold 7 was used. Array CGH profiles derived from two dye-swap experiments were plotted together on the same plot. Synteny between human and mouse chromosomes were analysed on the Ensembl Comparative Genomics/Synteny website. The ideograms of human chromosomes 13 and 20 and mouse chromosomes 2 and 14 shown in Fig. 4c were generated by tracing illustrations in the Ideogram View on the NCBI Genome Data Viewer website. Syntenic comparison of human and mouse chromosome was performed using Comparative Genomics/Synteny tool on Ensembl website.

\section{Mutation and copy number analysis using TCGA data}

Exome and SNP array data from TCGA COADREAD paired tumour/normal samples were prepared as described previously ${ }^{77}$ Briefly, mutation calling from the exome data was performed using the EBCall algorithm ${ }^{78}$, and absolute copy number profiles were obtained from the SNP array data using the ASCAT algorithm ${ }^{79}$. We analysed the copy numbers of $13 q$ and $20 \mathrm{q}$. For 20q, the AURKA locus alone, the TPX2 locus alone, and 20q containing both loci were analysed 
independently. We assumed that 13q, 20q, AURKA, or TPX2 had undergone chromosomal gain if the sum of the allele-specific copy numbers averaged along the loci was greater than or equal to three. Similarly, each of the loci was assumed to have undergone chromosomal loss if the total copy number was less than or equal to one. For the $A P C$ mutation, we analysed only patient samples carrying APC truncations within the region between the N-terminus and the SAMP repeats. Seven cases with $\beta$-catenin stabilizing mutations were omitted from the analysis. In total, 291 COAD and 111 READ TCGA patient samples were selected. The 402 patient samples were divided into three groups, as follows:

Class 1: Lesions with two or more (two to three as a result) truncating mutations in the $A P C$ locus, or one or more (one to three as a result) truncating mutations and $\mathrm{LOH}$ in the $A P C$ locus, which are expected to have no wild-type $A P C$.

Class 2: Lesions with one truncating mutation or one $\mathrm{LOH}$ in the $A P C$ locus, which are expected to have a single $A P C$ mutation and retain a single wild-type copy of $A P C$.

Class 3: Lesions without truncating mutation and $\mathrm{LOH}$ in the $A P C$ locus.

Among the three groups, copy number alterations of 13q, 20q, AURKA, and TPX2 were analysed using Chi-squared test (two-sided) and the Mann-Whitney $U$ test (two-sided). Because the results for 20q, AURKA, and TPX2 were almost same, only the 20q results are presented.

\section{Karyotyping of chromosomes in MCF10A and APC883 cells}

The APC833 cell line was generated from the MCF10A cell line, a genetically stable human mammary gland epithelial cell line ${ }^{80}$, by introducing a truncation mutation into the APC locus by genome editing. The MCF10A cell line was established in 1990 from human fibrocystic mammary tissue ${ }^{81}$. Karyotyping prior to immortalization showed normal diploidy, but later 
passages showed near-diploidy with some rearrangements interpreted as

$48, X X, 3 p-, 6 p+, 8+, 9 p+, 16+$, including a balanced reciprocal translocation $\mathrm{t}(3 ; 9)(\mathrm{p} 13 ; \mathrm{p} 22)$ and an unbalanced translocation $\mathrm{t}(5 \mathrm{q} ; \operatorname{der}[9])^{81}$ In later studies, gain at chromosome $1 \mathrm{q}$ was reported ${ }^{82,83}$, as observed in this study. Trisomy 20 is recurrent in subsequent studies $^{82-85}$,

In this study, Q-banding (20 cells for each cell type) were performed by Chromosome Science Labo (Hokkaido, Japan). Complete description of karyotyping results is shown in Table 1. In our early analysis, detailed rearrangement of one chromosome 8 was not determined and was described as “dup8(p?)” In later analysis, the structure of the rearranged chromosome 8 was analyzed by FISH using specific probes (8p11, 8p23, 8q13, 8q21, 8q24) and the description was updated to "der(8)dup(8)(q22q24.3)ins(8)(p24.3q22q24.3)". For the transformed, near-tetraploid APC883 cells, common number imbalances are shown by letter color for karyotype description: cyan, three chromosome 6s; brown, five chromosome 8s; red, six chromosome 20s (Table 1), and by box color for chromosome images: cyan, three chromosome $6 \mathrm{~s}$; brown, five chromosome 8s; red, six chromosome 20s. To analyze newly generated chromosome aberrations, types of abnormalities were classified as tetraploidy, gain, loss, deletion and fusion. The fusion includes all types of chromosomes generated by fusions such as translocation, robertsonian translocation and telomere association.

\section{FISH analysis of mouse intestine tissues}

BAC clones containing mosue Aurka (B6Ng01-332M20) and Col5al (B6Ng01-295J05) genes were obtained from RIKEN BRC DNA Bank. BAC DNAs were labeled with Alexa Fluor 488 dye (F32947) or Alexa Fluor 555 dye (F32947) using FISH Tag DNA Kit (Thermo Fisher Scientific) by nick-translation according to the manufacturer's instructions. Paraffin sections of 
intestinal tissues from an 16-weeks old $A p c^{\Delta 716}$ mouse were processed through deparaffinization and rehydration using standard procedures. The slides were treated with Spot-Light Tissue Pretreatment Kit (Thermo Fisher Scientific, 008401) and labeled with FISH proves according to the manufacturer's recommendations. The labeled slides were treated with Vector TrueVIEW Autofluorescence Quenching Kit (Vector Laboratories, Burlingame, CA, US) and mounted in Vectashield (Vector Laboratories) according to manufacturer's instructions. The specimens were imaged using an Imager Z1 fluorescence microscope (Carl Zeiss).

\section{Statistics and reproducibility}

Statistical evaluation was carried out by Student's t-test (two-sided), Chi-squared test (twosided), Tukey-Kramer method (two-sided), F-test (two-sided) or Mann-Whitney U test (twosided) as indicated. Data are shown as means \pm SEM. P-values and sample size are shown in each Figure. Biochemical experiments were repeated in at least three independent preparations with essentially similar results. For quantification of image data, to ensure adequate power to detect significance, sufficient numbers of regions/cells were analysed as indicated in each Figure.

\section{Data and code availability}

Data and computer codes are available on request from the authors. Materials used in this study are available for distribution following MTA, except for materials from human research participants. The aCGH data discussed in this publication have been deposited in NCBIs Gene Expression Omnibus (GEO; http://www.ncbi.nlm.nih.gov/geo/) and are accessible through GEO 
series accession number GSE3642. accession GSE63306

(https://www.ncbi.nlm.nih.gov/geo/query/acc.cgi?acc= GSE63306).

\section{Reference}

64. Mimori-Kiyosue, Y. et al. CLASP1 and CLASP2 bind to EB1 and regulate microtubule plus-end dynamics at the cell cortex. The Journal of cell biology 168, 141-153 (2005).

65. Nakamura, S. et al. Dissecting the nanoscale distributions and functions of microtubuleend-binding proteins EB1 and ch-TOG in interphase HeLa cells. PloS one 7, e51442 (2012).

66. Shirayoshi, Y., Nose, A., Iwasaki, K. \& Takeichi, M. N-linked oligosaccharides are not involved in the function of a cell-cell binding glycoprotein E-cadherin. Cell Struct Funct $11,245-252$ (1986).

67. Hotta, A. et al. Laminin-based cell adhesion anchors microtubule plus ends to the epithelial cell basal cortex through LL5alpha/beta. The Journal of cell biology 189, 901-917 (2010).

68. Kawasaki, Y. et al. Asef, a link between the tumor suppressor APC and G-protein signaling. Science 289, 1194-1197 (2000).

69. Takahashi, N., Ishihara, S., Takada, S., Tsukita, S. \& Nagafuchi, A. Posttranscriptional regulation of alpha-catenin expression is required for Wnt signaling in L cells. Biochem Bioph Res Co 277, 691-698 (2000).

70. Yamamoto, H. et al. Axil, a member of the Axin family, interacts with both glycogen synthase kinase 3 beta and beta-catenin and inhibits axis formation of Xenopus embryos. Mol Cell Biol 18, 2867-2875 (1998).

71. Oshimori, N., Ohsugi, M. \& Yamamoto, T. The Plk1 target Kizuna stabilizes mitotic centrosomes to ensure spindle bipolarity. Nature cell biology 8, 1095-1101 (2006).

72. Pronobis, M.I., Deuitch, N., Posham, V., Mimori-Kiyosue, Y. \& Peifer, M. Reconstituting regulation of the canonical Wnt pathway by engineering a minimal beta-catenin destruction machine. Molecular biology of the cell 28, 41-53 (2017).

73. Hino, S., Tanji, C., Nakayama, K.I. \& Kikuchi, A. Phosphorylation of beta-catenin by cyclic AMP-dependent protein kinase stabilizes beta-catenin through inhibition of its ubiquitination. Mol Cell Biol 25, 9063-9072 (2005). 
74. Oshima, H., Oshima, M., Kobayashi, M., Tsutsumi, M. \& Taketo, M.M. Morphological and molecular processes of polyp formation in Apc(Delta 716) knockout mice. Cancer research 57, 1644-1649 (1997).

75. Yokoyama, A. et al. The C-terminal domain of the adenomatous polyposis coli (Apc) protein is involved in thyroid morphogenesis and function. Medical molecular morphology 44, 207-212 (2011).

76. Sells, T.B. et al. MLN8054 and Alisertib (MLN8237): Discovery of Selective Oral Aurora A Inhibitors. ACS medicinal chemistry letters 6, 630-634 (2015).

77. Uchi, R. et al. Integrated Multiregional Analysis Proposing a New Model of Colorectal Cancer Evolution. PLoS genetics 12, e1005778 (2016).

78. Shiraishi, Y. et al. An empirical Bayesian framework for somatic mutation detection from cancer genome sequencing data. Nucleic acids research 41, e89 (2013).

79. Van Loo, P. et al. Allele-specific copy number analysis of tumors. Proceedings of the National Academy of Sciences of the United States of America 107, 16910-16915 (2010).

80. Debnath, J., Muthuswamy, S.K. \& Brugge, J.S. Morphogenesis and oncogenesis of MCF$10 \mathrm{~A}$ mammary epithelial acini grown in three-dimensional basement membrane cultures. Methods 30, 256-268 (2003).

81. Soule, H.D. et al. Isolation and characterization of a spontaneously immortalized human breast epithelial cell line, MCF-10. Cancer research 50, 6075-6086 (1990).

82. Cowell, J.K. et al. Molecular characterization of the t(3;9) associated with immortalization in the MCF10A cell line. Cancer genetics and cytogenetics 163, 23-29 (2005).

83. Jonsson, G. et al. High-resolution genomic profiles of breast cancer cell lines assessed by tiling BAC array comparative genomic hybridization. Genes, chromosomes \& cancer 46, 543-558 (2007).

84. Zientek-Targosz, H. et al. Transformation of MCF-10A cells by random mutagenesis with frameshift mutagen ICR191: a model for identifying candidate breast-tumor suppressors. Molecular cancer 7, 51 (2008).

85. Marella, N.V. et al. Cytogenetic and cDNA microarray expression analysis of MCF10 human breast cancer progression cell lines. Cancer research 69, 5946-5953 (2009).

86. Aoki, K. \& Taketo, M.M. Adenomatous polyposis coli (APC): a multi-functional tumor suppressor gene. J Cell Sci 120, 3327-3335 (2007). 
87. Polakis, P. The adenomatous polyposis coli (APC) tumor suppressor. Biochimica et biophysica acta 1332, F127-147 (1997).

88. Pronobis, M.I., Rusan, N.M. \& Peifer, M. A novel GSK3-regulated APC:Axin interaction regulates Wnt signaling by driving a catalytic cycle of efficient betacatenin destruction. eLife 4, e08022 (2015).

89. Näthke, I.S. The adenomatous polyposis coli protein: the Achilles heel of the gut epithelium. Annual review of cell and developmental biology 20, 337-366 (2004).

90. Powell, S.M. et al. APC mutations occur early during colorectal tumorigenesis. Nature 359, 235-237 (1992).

91. Horii, A. et al. The APC gene, responsible for familial adenomatous polyposis, is mutated in human gastric cancer. Cancer research 52, 3231-3233 (1992).

92. Boynton, R.F. et al. Loss of heterozygosity involving the APC and MCC genetic loci occurs in the majority of human esophageal cancers. Proceedings of the National Academy of Sciences of the United States of America 89, 3385-3388 (1992).

93. Joslyn, G. et al. Identification of deletion mutations and three new genes at the familial polyposis locus. Cell 66, 601-613 (1991).

94. Groden, J. et al. Identification and characterization of the familial adenomatous polyposis coli gene. Cell 66, 589-600 (1991).

95. Miyoshi, Y. et al. Somatic mutations of the APC gene in colorectal tumors: mutation cluster region in the APC gene. Human molecular genetics 1, 229-233 (1992).

96. Su, L.K. et al. Multiple intestinal neoplasia caused by a mutation in the murine homolog of the APC gene. Science 256, 668-670 (1992).

97. Moser, A.R., Pitot, H.C. \& Dove, W.F. A dominant mutation that predisposes to multiple intestinal neoplasia in the mouse. Science 247, 322-324 (1990).

\section{Acknowledgements}

We are grateful to Y. Tano (Riken BDR) for her assistance in experiments and data processing.

We are grateful also to Dr. Y. Ono (Riken BDR) for his instruction of FISH analysis, Drs. A.

Nagafuchi (Nara Medical Univ), T. Toda (Hiroshima Univ), K. Yamagata (Kindai Univ), M. 
Toya (Waseda Univ), T. Kitajima (Riken BDR), M. Takeichi (Riken BDR), S. Kuratani (Riken BDR), and Y. Takai (Kobe Univ) for critical reading of the manuscript and valuable suggestions.

Our special thanks are also due to Ms. M. Yamaguchi (Carl Zeiss Japan) and Mr. M. Yamaguchi (Molecular Devices Japan) for excellent technical assistance in operating the microscope systems and image analysis software. Y.M.-K. was supported by the Japan Society for the Promotion of Science-NEXT program (LS128), the Takeda Science Foundation, the Uehara Memorial Foundation, a Grant-in-Aid for Challenging Exploratory Research (Japan Society for the Promotion of Science KAKENHI no. 20K20379), and Japan Science and Technology Agency Core Research for Evolutional Science and Technology (no. JPMJCR1863), Extramural Collaborative Research Grant of Cancer Research Institute, Kanazawa University, and an intramural grant from RIKEN. The results published here are in part based upon data generated by the TCGA Research Network: http://cancergenome.nih.gov/.

\section{Author contributions}

Y.K., T.H. K.O. A.H. and T.N. performed experiments and edited the manuscript. Y.W. and M.O. performed experiments. Y.K., A.N., and K.M. analyzed TCGA data using informatics techniques. T.K., T.S., M.O., K.F., A.K., P.O W. and H.O. prepared biological samples. K.K. performed image data analysis and statistical data analysis. N.Y., M.M., H.Y., C.W.X., S.A., K.T., W.R.L., B-C.C. and E.B. performed imaging experiments, image processing and data analysis. R.S. and R.F. prepared biological samples and edited the manuscript. M.O., M.M.T and T.A gave conceptual and technical advices. Y.M.-K. conceived and managed the project, performed experiments, and wrote the manuscript. 


\section{Competing interests}

Portions of the technology described herein are covered by U.S. Patent 7,894,136 issued to Eric Betzig (EB) and assigned to Lattice Light, LLC of Ashburn, VA, U.S. Patents 8,711,211 and 9,477,074 issued to EB and assigned to HHMI, U.S. Patent application 13/844,405 filed by EB and Kai Wang (KW) and assigned to HHMI, and U.S. Patent 9,500,846 issued to EB and KW and assigned to HHMI. 
Figure S1 Kawasaki et al.

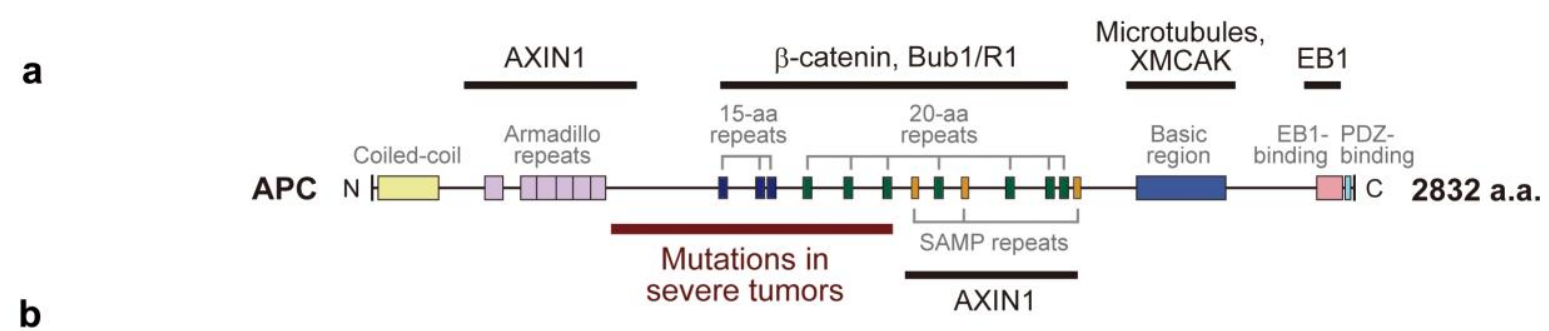

APC truncating mutations

$A p c^{1638 T}$ mouse

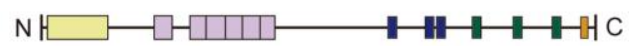

$A p c^{\text {Min }}$ mouse

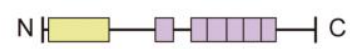

$A p c^{\triangle 716}$ mouse

APC883 cell line
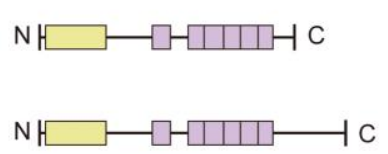

Phenotype

Heterozygous Homozygous

Tumor free

Severe intestinal tumor after $\mathrm{LOH}$

Tumor free, weaker CIN in ES cells

Severe intestinal tumor after $\mathrm{LOH}$

Embryonic lethal, severe CIN in ES cells

N.D.

Embryonic lethal

This study

Fig. S1 APC structure and phenotypes. (a) Domain structures of full length APC and major binding partners discussed in this study ${ }^{29,40,41,86-89}$. The APC gene is responsible for many sporadic cases of gastrointestinal cancers and familial adenomatous polyposis (FAP), a dominantly inherited colorectal tumour predisposition that results from germline mutations in $\mathrm{APC}^{21,22,90-94}$. Tumours start developing upon mutation in both alleles. In general, APC mutations that result in stable proteins truncated in the region between the armadillo repeat and first SAMP motif are associated with severe polyposis phenotypes (reviewed in 40, 95). (b) Truncated $A P C$ mutants. Phenotypes of cells and mice are shown on the right. Heterozygous Apc mutations, such as $A p c^{M i n}$ and $A p c^{4716}$, leads to severe polyposis after loss of the wild-type allele (> 30 polyps), whereas their homozygosity results in embryonic lethality ${ }^{46,96,97}$. Homozygous $A p c^{1638 T}$ mice harboring a truncating mutation just after the first SAMP motif, which retains the $\beta$-catenin destruction ability, are viable and do not develop any tumours, demonstrating that this 
N-terminal fragment retaining the first SAMP motif is sufficient for the tumour-suppressing function $^{39}$. Nevertheless, $A p c^{1638 T}$ homozygous ES cells present a CIN phenotype, although it is weaker than that in $A p c^{\text {Min }}$ homozygous ES cells ${ }^{16}$. MCF10A-derived cell line APC883

harbouring a homozygous APC truncation was generated by genome editing. 
Figure S2 Kawasaki et al.

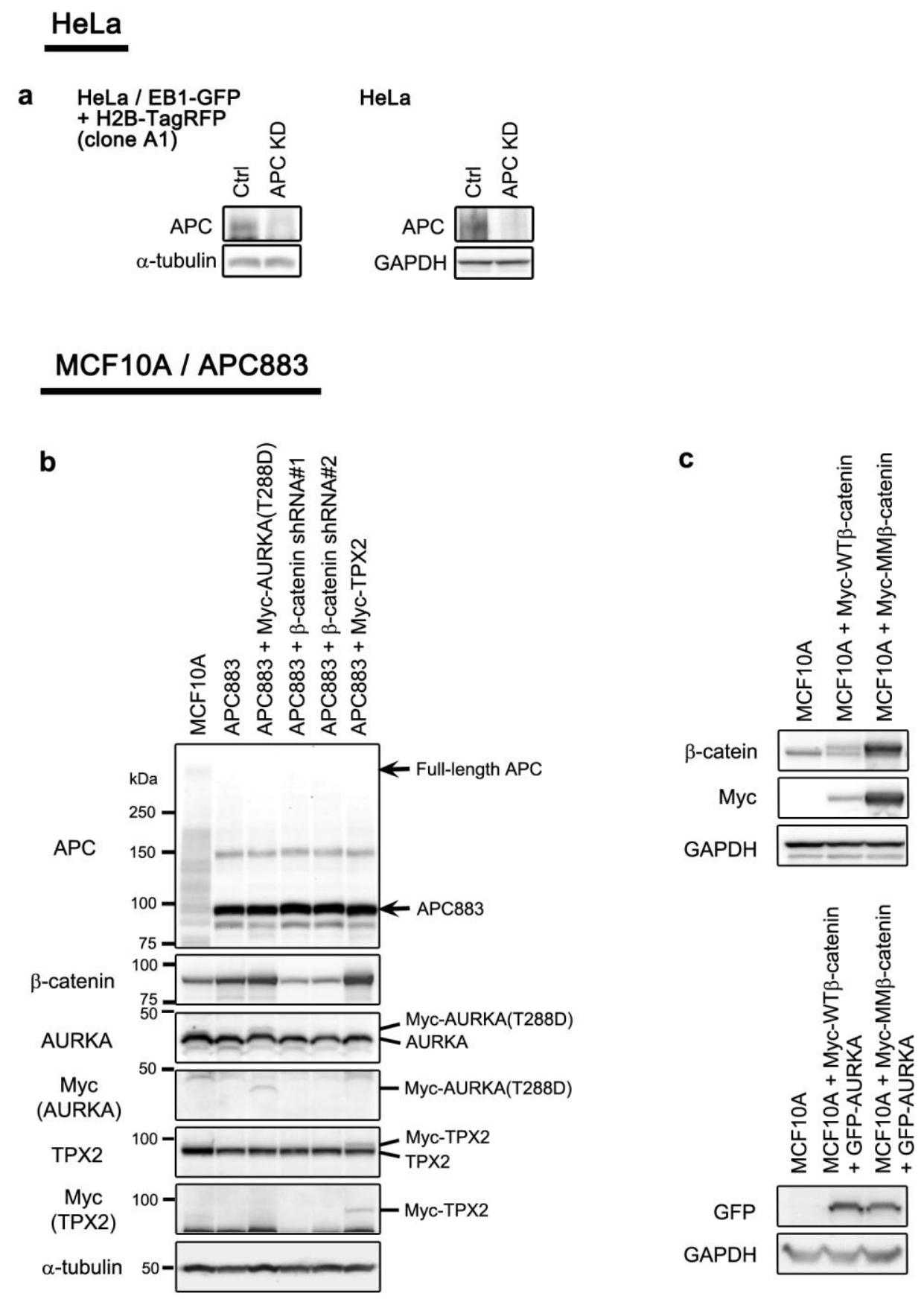

Fig. S2 Characterization of cells used in this study. (a) Western blot analysis of HeLa cells and HeLa cells expressing EB1-GFP and H2B-TagRFP (clone A1) ${ }^{31}$ before and after constitutive 
transfection with shRNA against APC (APC KD). APC knockdown efficiency was 70\%. (b) Characterization of MCF10A cells, APC883 cells, and APC883 cell-derived transfectants. Cell lysates were subjected to western blotting using the indicated antibodies. Full length APC and truncated APC883 protein are indicated. For Myc-AURKA (T288D) and Myc-TPX2, endogenous and exogenous Myc-tagged proteins are indicated. (c) Characterization of MCF10A cells transfected with Myc-tagged wild-type $\beta$-catenin (Myc-WT $\beta$-catenin) and a dominant stable $\beta$-catenin (Myc-MM $\beta$-catenin), and further transfected with GFP-AURKA for fluorescence recovery after photobleaching (FRAP) analysis. Myc-WT $\beta$-catenin- and MycMM $\beta$-catenin-transfected cells were established under the same conditions, but Myc-WT $\beta$ catenin was hardly overexpressed. 


\section{Figure S3 Kawasaki et al.}
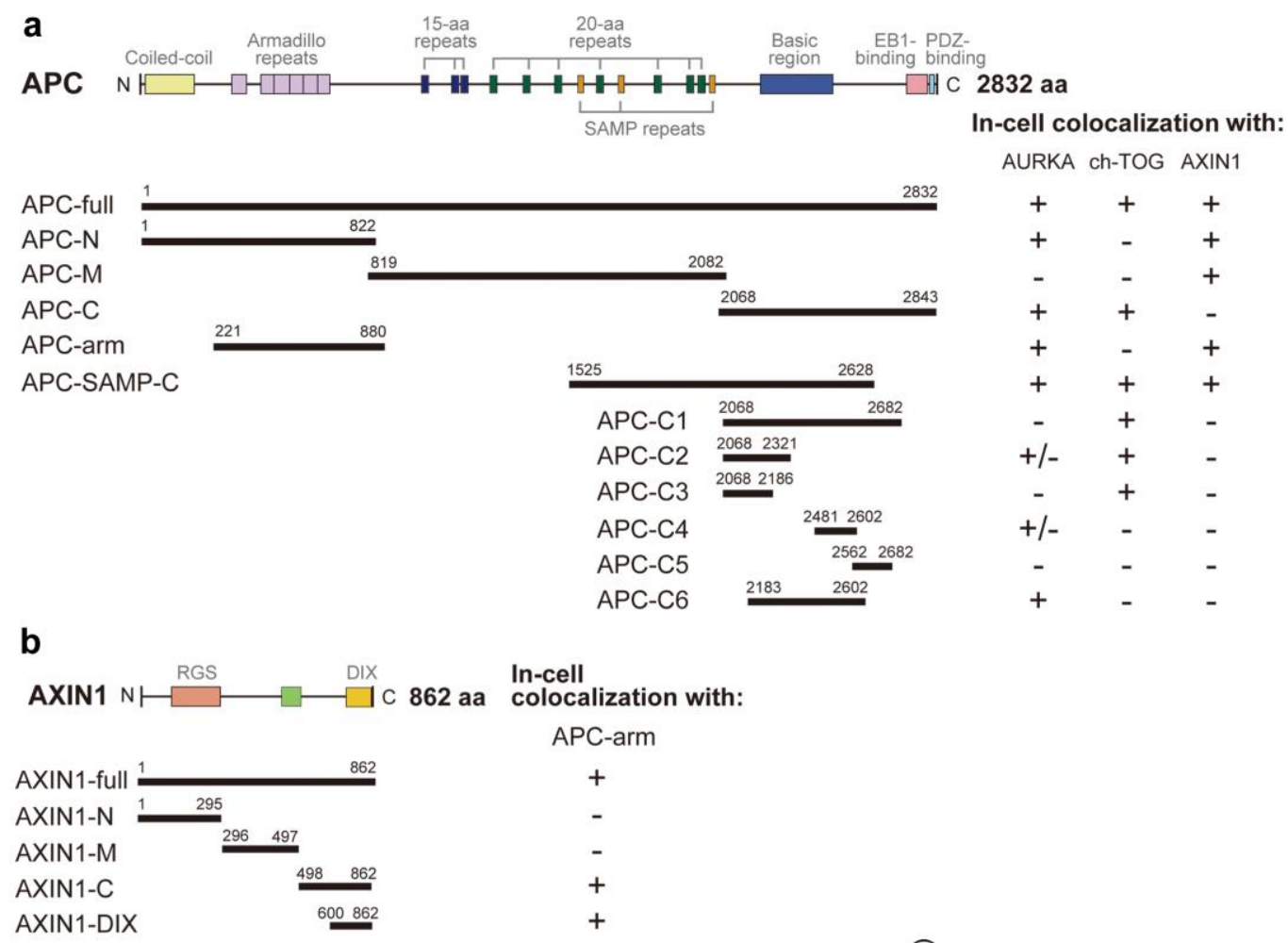

b

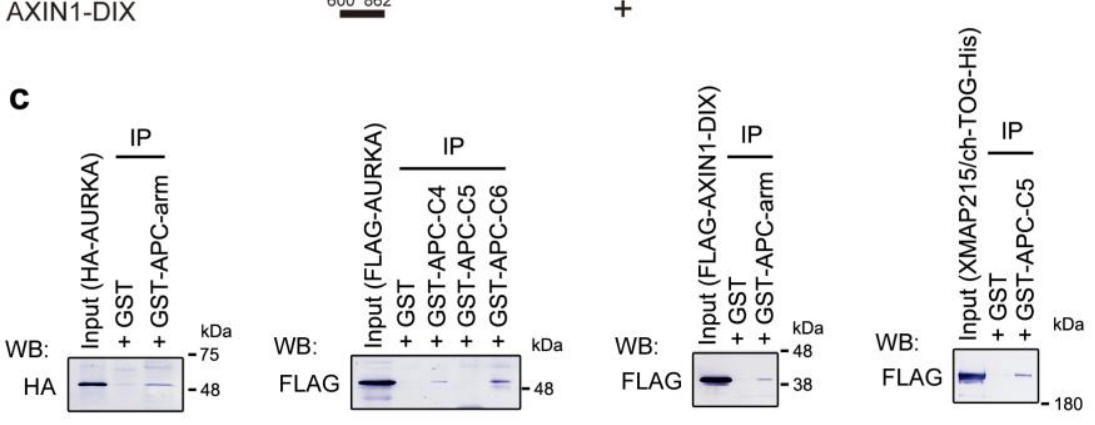

d

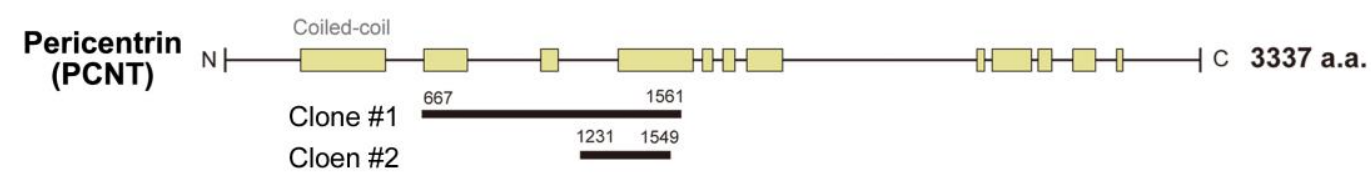

Fig. S3 Identification of APC-binding proteins. (a, b) Domain structures of APC and AXIN1, and the analyzed fragments are shown. Amino acid numbering is based on human APC transcript variant 3 (NM_000038) and human AXIN1 transcript variant 1 (NM_003502). Associations of 
APC with AURKA and AXIN1 were analyzed by in-cell colocalization analysis using the series of deletion mutants. The results are shown on the right. (c) Direct binding between APC fragments and AURKA/AXIN1/ch-TOG predicted in (a) was confirmed by in vitro pull-down assays using purified proteins. The APC arm and APC-C6 bound to AURKA. The APC arm also bound to the C-terminal DIX domain of AXIN1. XMAP215, the Xenopus homologue of chTOG, bound to APC-C3. We used Xenopus XMAP215 because we could not purify full length human ch-TOG from E. Coli. The C-terminal one-third bound to the APC arm. (d) Yeast twohybrid screening using the APC arm region as bait identified pericentrin. The two identified pericentrin clones are shown. 
Figure S4 Kawasaki et al.

a

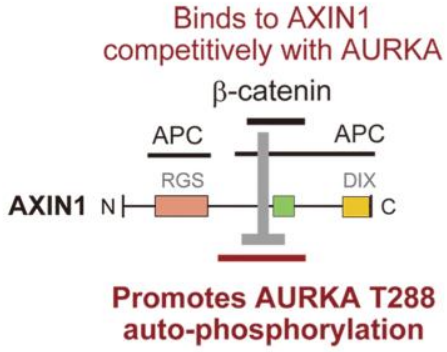

b

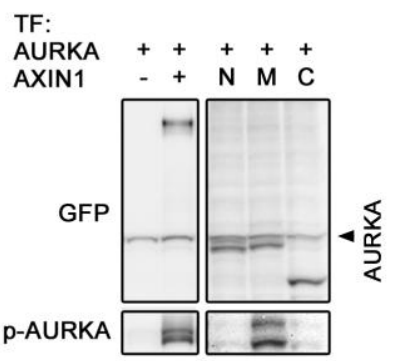

C
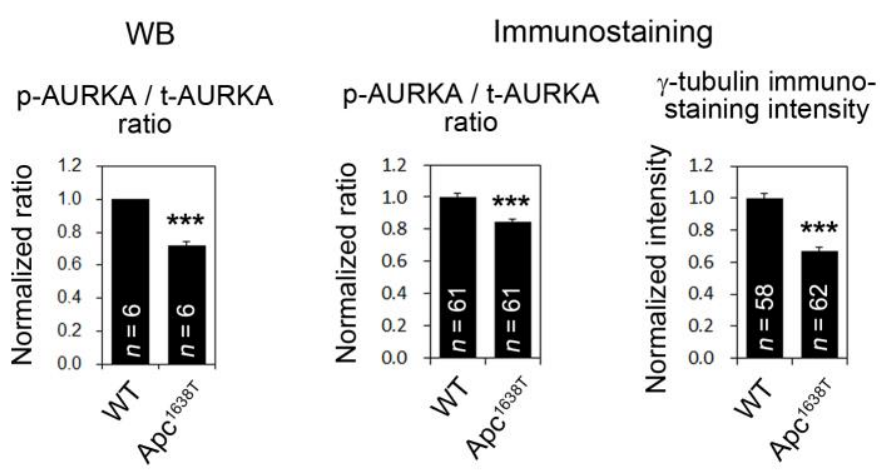

Fig. S4 Biochemical analysis of AURKA regulation by APC and AXIN1. (a) AXIN1

structure and AURKA-activating region that also binds to $\beta$-catenin ${ }^{37}$. (b) Effects of AXIN1 on AURKA phosphorylation were analyzed using the HEK293T cell overexpression assay system. HEK293T cells were transfected with GFP-fused AURKA and full length AXIN1 or fragments, lysed, and then subjected to western blotting using anti-GFP and anti-p-T288 AURKA antibodies. (c) Analysis of primary MEFs from wild-type (WT) and $A p c^{1638 T}$ mice. WB analysis of phospho-to-total AURKA ratio, and the immunostaining intensity of phospho-to-total AURKA ratio and $\gamma$-tubulin at centrosomes were normalized to those of the wild-type, which were set to $1.0(\mathrm{c})$. Data are shown as means $\pm \mathrm{SE}$; ***P $<0.001$; Student's t-test. 
Figure S5 Kawasaki et al.

\section{a MCF10A}

Type No.1: Chromosome 20 trisomy

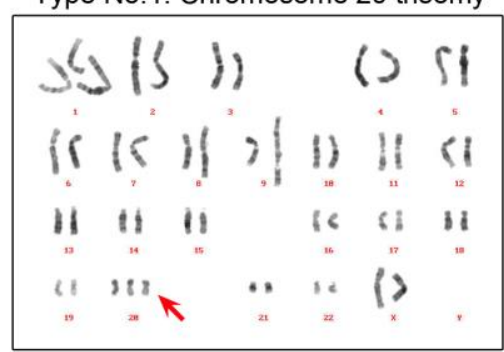

b $\mathrm{APC} 883$

Type No.3: Dicentric derivative chromosome containing chrmosome 7 and 9

\begin{tabular}{|c|c|c|c|c|c|c|}
\hline$f^{\prime}$ & $\beta$ & & & $t_{m}^{1}$ & i) & if \\
\hline 13 & 8 & if & i & it & 18 & 11 \\
\hline . & , & " & , & "' & " & $" \mathrm{z}$ \\
\hline 18 & 60 & 18 & & 88 & 18 & $\mathrm{ab}$ \\
\hline " & " & 15 & & " & $"$ & "' \\
\hline 11 & 88 & & 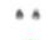 & $\theta 0$ & 57 & \\
\hline$"$ & "m & & a & ${ }^{x}$ & ${ }^{*}$ & r \\
\hline
\end{tabular}

e $\mathrm{APC} 883+\mathrm{TPX} 2$

Type No.3: Additional material of unknown origin on chromosome 8

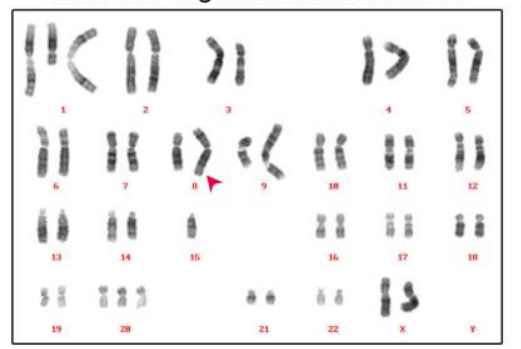

Type No.5: Derivative chromosomes containing chromorome 1 , and 6 and 14

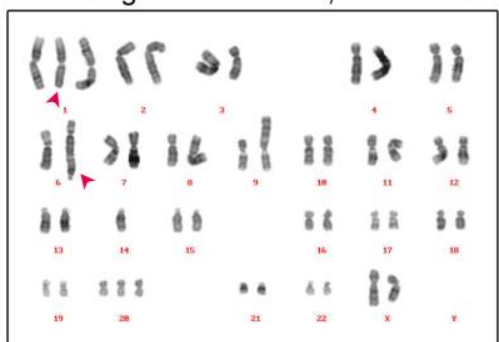

Type No.2: Chromosome 20 disomy

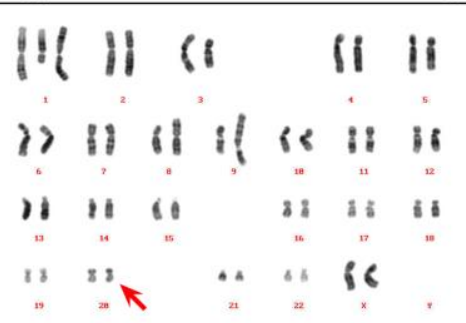

C

APC883 + $\beta$-catenin shRNA\#1 Type No.1: Original karyotype

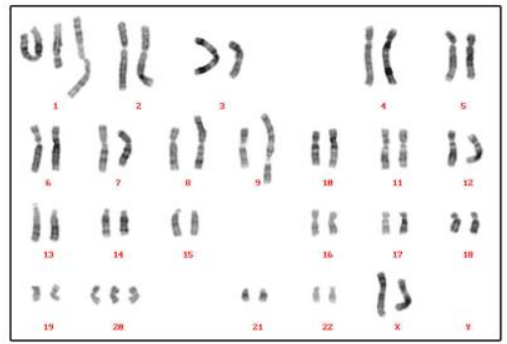

d

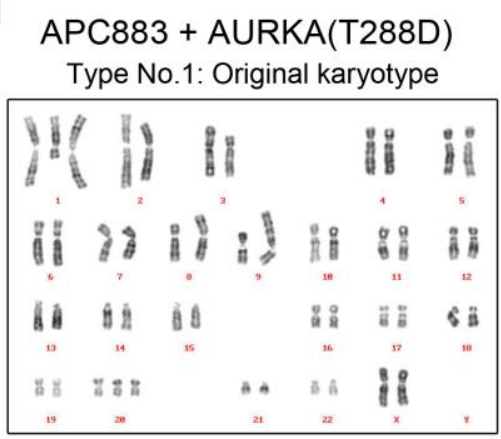

Type No.5: Deletion of chromosome 1 and 12 , and $1 ; 12$ translocation

\begin{tabular}{|c|c|c|c|c|c|c|}
\hline 61 & 1 & 1 & 1 & & il & ii \\
\hline I & $=$ & & , & & . & " \\
\hline if & If & i) & i: & है & 28 & is \\
\hline - & r & " & , & 10 & " & 12 \\
\hline 18 & it & 10 & & i: & ä & î \\
\hline & "s & & & & & \\
\hline 88 & 98 & & ob & Ab & 82 & \\
\hline
\end{tabular}

f Near-tetraploid APC883

Type No.1: Near-tetraploid

\begin{tabular}{|c|c|c|c|c|c|c|}
\hline है है है & E\& & คำดีด & 배에 & ถ้ำดำ & 19:18 & בด \\
\hline OB A A A & 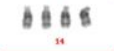 & if ef it & & 328 & 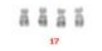 & 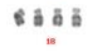 \\
\hline 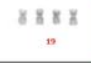 & $\begin{array}{c}288828 \\
7\end{array}$ & & 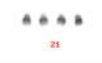 & 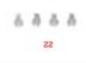 & 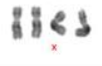 & $v$ \\
\hline
\end{tabular}


analyzed cells, see Table 1. The karyotypes were determined by Q-banding with Hoechst/quinacrine staining. Note that commonly observed abnormalities in chromosome 1 (deletion and partial duplication), chromosome 3 (deletion and translocation), chromosome 8 (duplication), and chromosome 9 (translocation) have been observed previously ${ }^{81,82}$. (a) Karyotype of parental MCF10A cells expressing full length APC. The MCF10A cell line used has two major populations with or without chromosome 20 trisomy (types 1 and 2, respectively), whereas all 20 cells showed near-diploidy. Chromosome 20 is indicated by red arrows. (b-f) Karyotypes of APC883 cells and APC883 cell-derived cells. Major karyotypes in each cell type (c, d) and representative examples carrying chromosome aberrations (b, e, and f) are shown. Newly derived abnormal chromosomes are indicated by red arrowheads. After reduction of $\beta$ catenin levels (c) or expression of constitutively active AURKA (T288D) (d), APC883 cells maintained their original karyotype. In TPX2-expressing APC883 cells (e), various aberrations were observed. In (f), representative karyotypes of APC883 cells after > 5 months passages are shown. In this subpopulation, all cells analyzed had near-tetraploid karyotype with some numerical imbalances. Common numerical imbalances are shown by the letter color for the karyotype description: cyan, three chromosome 6s; brown, five chromosome 8s; red, six chromosome 20s. Examples of randomly occurring imbalances are indicated by arrows. 
Figure S6 Kasawaki et al.

a $A p c^{\Delta 716}$ mouse intestine

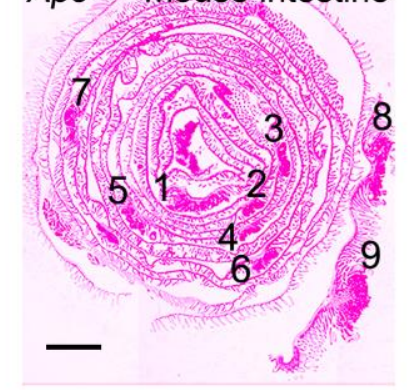

b
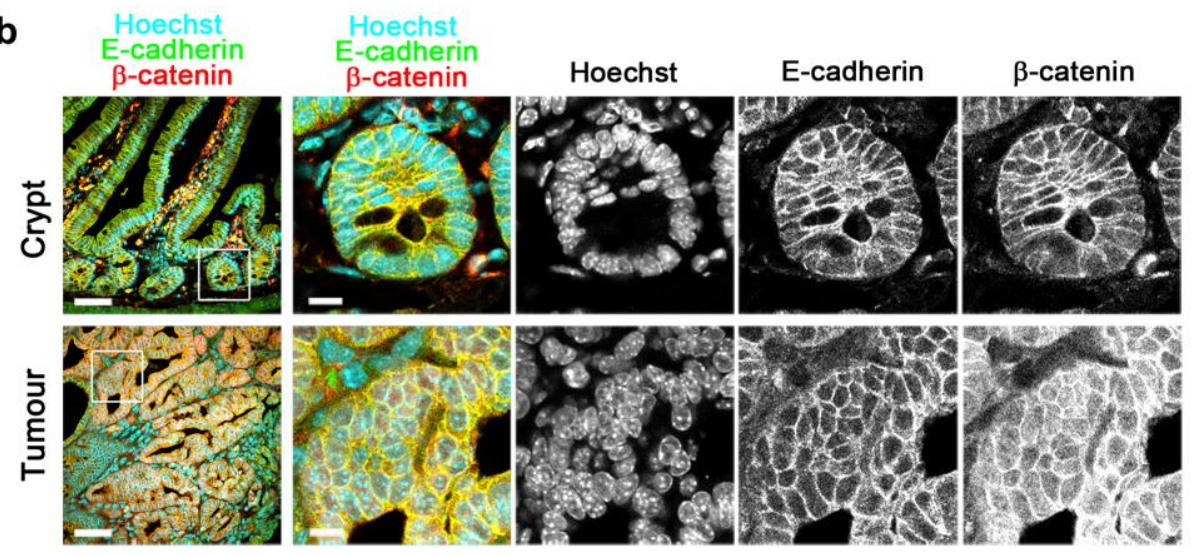

c

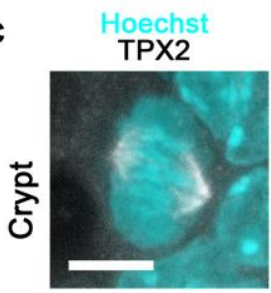

Hoechst

TPX2
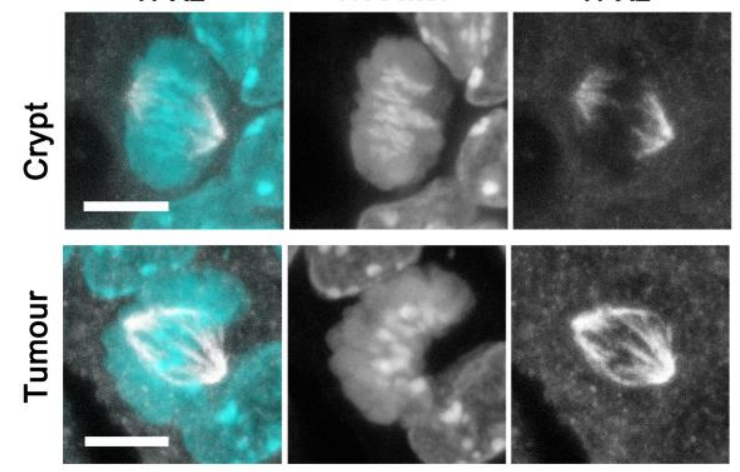

Fig. S6 Immunofluorescence analysis of paraffin-embedded sections of APC mutant mouse

intestines. (a) HE-stained section of the intestines of an 16-weeks old $A p c^{4716}$ mouse. Polyps analyzed in this study are indicated by numbers. (b) Immunostaining of a formalin-fixed, paraffin-embedded $A p c^{4716}$ mouse intestine tissue section for E-cadherin and $\beta$-catenin with 
nuclear DNA stained by Hoechst 33342 to visualize crypt cells (top) and tumour cells (bottom) is shown. Low magnification images are shown on the left, and magnified images of the boxed areas are shown in the four panels on the right. Note that $\beta$-catenin levels are upregulated but its preferential accumulation to the nucleus is not observed. (c) Upregulation of TPX2 in $A p c^{4716}$ tumour tissues. Immunostaining of a paraffin-embedded section for TPX2 with nuclear DNA stained by Hoechst 33342 is shown. Crypt cells (top) and tumour cells (bottom) were observed. Scale bars: $50 \mu \mathrm{m}$ (b, low mag, left), $10 \mu \mathrm{m}$ (b, high magnification, right), and $5 \mu \mathrm{m}$ (c). 
Figure S7 Kawasaki et al.

a Human colon polyp \#1

(Hyperplasia polyp)

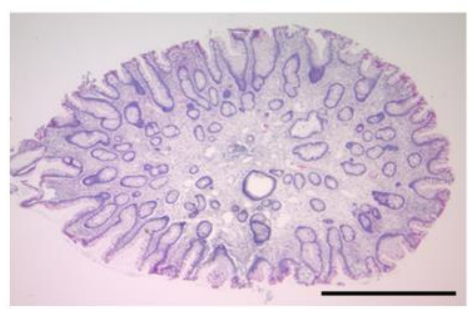

b $\begin{gathered}\text { Hoechst Ki67 } \\ \text { E-cadherin }\end{gathered}$

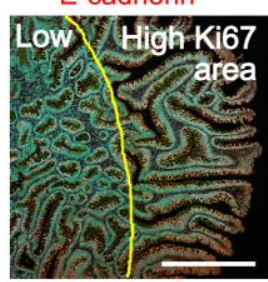

c
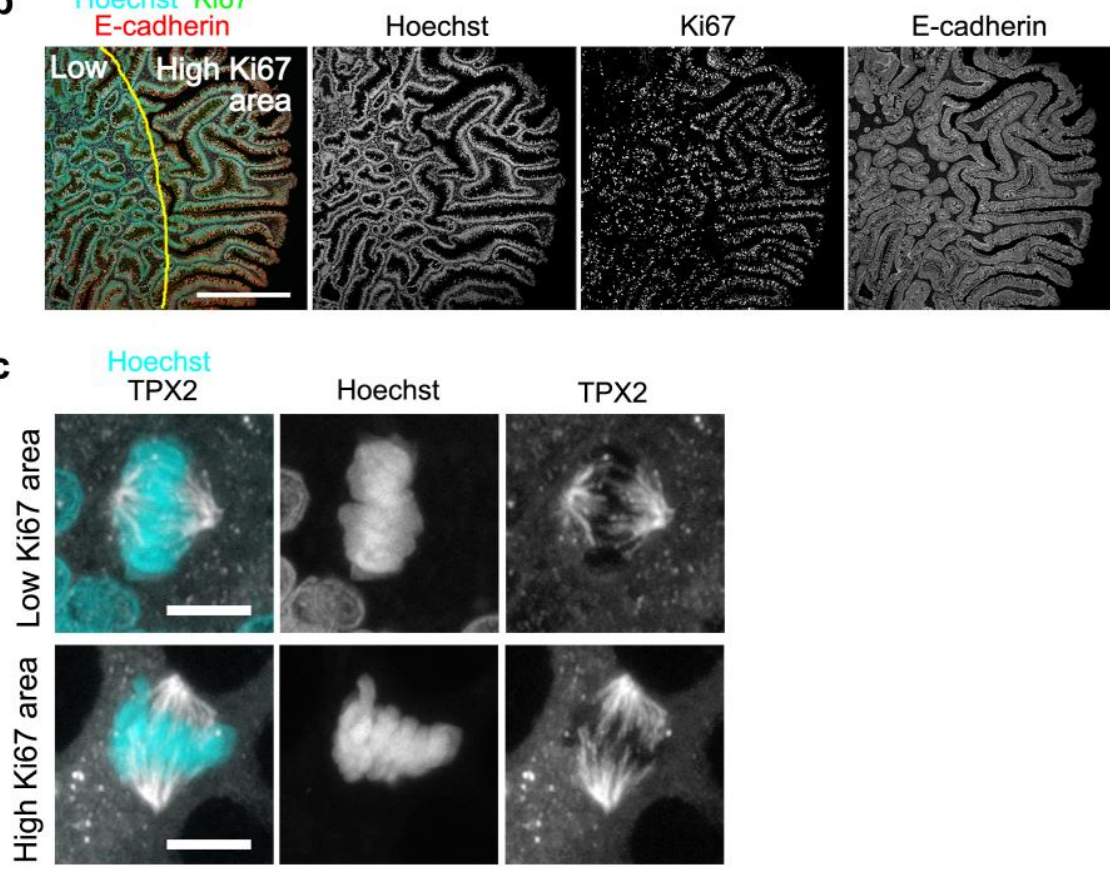

Human colon polyp \#2

(Low-grade tubular adenoma)
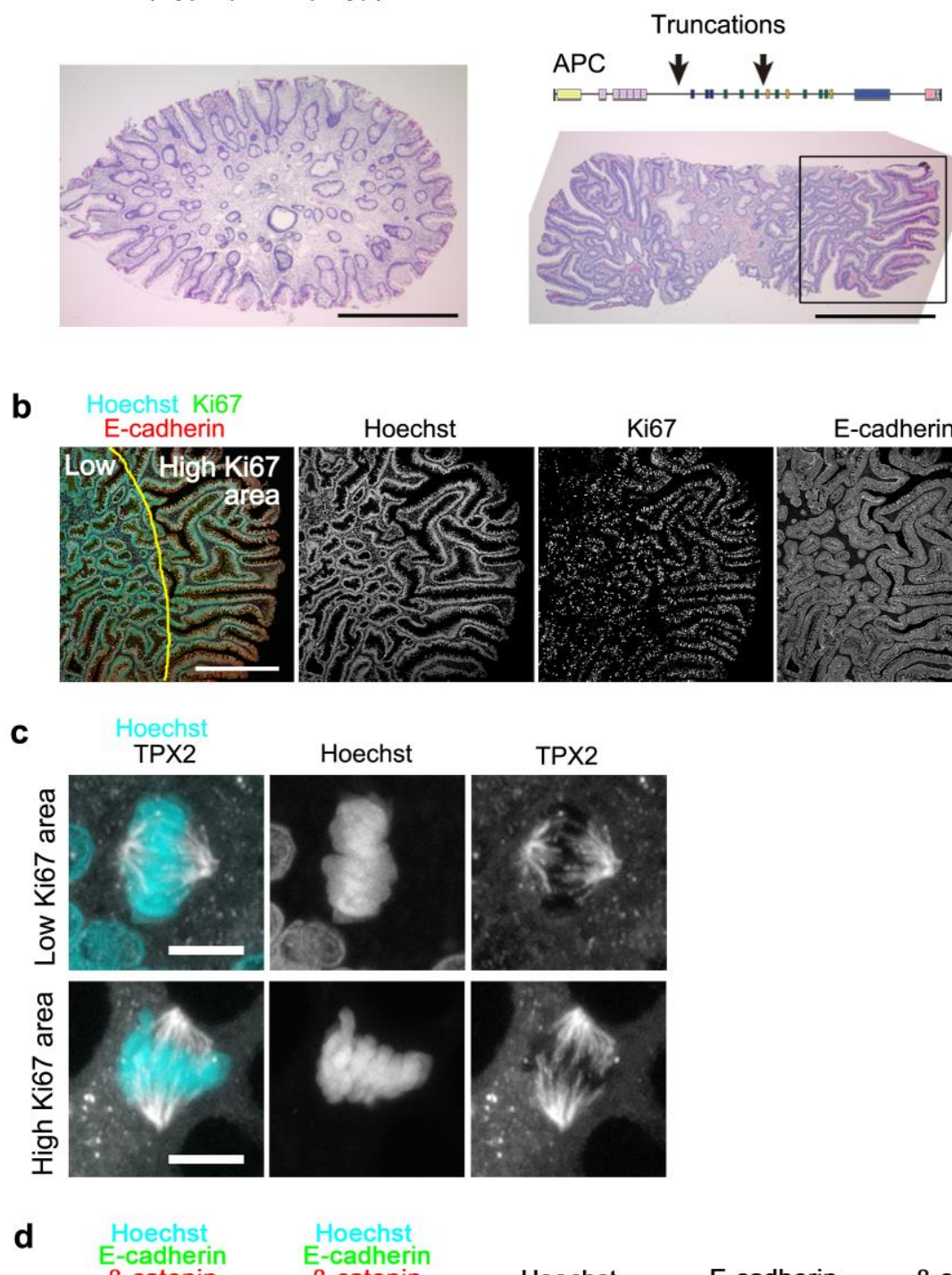

E-cadherin

$\beta$-catenin
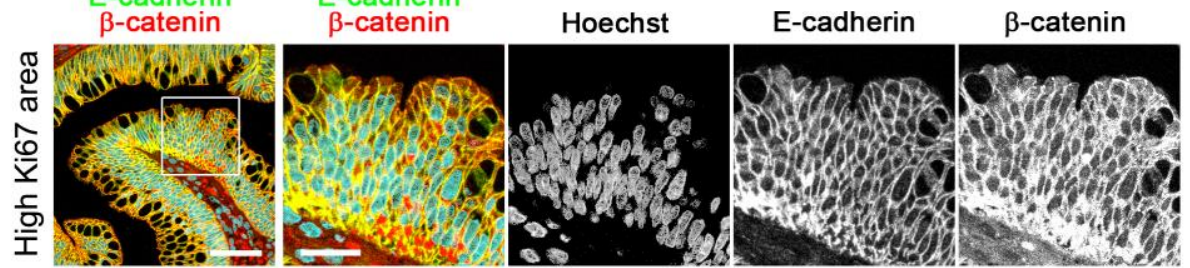

Fig. S7 Immunofluorescence analysis of paraffin-embedded sections of APC mutant

human tumor tissues. (a) HE-stained sections of a human hyperplasia polyp without APC 
mutation (polyp \#1) (left) and a low grade tubular adenoma containing two APC truncation mutations (polyp \#2) (right). Two mutant APC sequences detected in polyp \#2 are shown (arrows). For details, see also SI Materials and Methods. (b) Ki67 proliferation index of the boxed area in (a) in the polyp \#2. Immunofluorescence staining of polyp \#2 for E-cadherin and Ki67. Nuclear DNA was stained with Hoechst 33342. Based on the Ki67 intensity, the area was roughly divided into a highly proliferative region (outer, high Ki67 area) and a moderately proliferative region (inner, low Ki67 area) as indicated by the yellow line. (c) Immunofluorescence staining of polyp \#2 for TPX2 with Hoechst 33342 is shown. Examples of mitotic cells in less proliferative area (low Ki67) and highly proliferative area (high Ki67) shown in (b). (d) Immunostaining of the polyp \#2 for E-cadherin and $\beta$-catenin with nuclear DNA stained by Hoechst 33342 is shown. Low magnification images are shown on the left, and magnified images of the boxed areas are shown in the four panels on the right. Note that preferential accumulation of $\beta$-catenin to the nucleus is not observed. Scale bars: $1 \mathrm{~mm}(\mathrm{a}), 200$ $\mu \mathrm{m}(\mathrm{b}), 5 \mu \mathrm{m}$ (c), $50 \mu \mathrm{m}$ (d, left) and $20 \mu \mathrm{m}$ (d, right). 


\section{Table Legends}

\section{Table 1 (separate EXCEL file)}

This table contains full descriptions of the karyotypes of MCF10A, APC883, and transfectants. Newly generated chromosomes, including truncation and chromosome rearrangements, such as translocation, duplication, and telomere association, are shown in bold. For near tetraploid APC883 cells, the common number imbalance of chromosomes 6,8 , and 20 is indicated by color codes identical to the box colors in Fig. S5: cyan, three chromosome 6s; brown, five chromosome 8s; red, six chromosome 20s.

\section{Movie Legends}

\section{Movie 1}

3D time-lapse images of a mitotic HeLa cell expressing EB1-GFP and H2B-TagRFP collected by lattice light-sheet microscopy at $10 \mathrm{~s}$ intervals are represented in a three-dimensional space. Time scale: h.m.s.

\section{Movie 2}

3D time-lapse images of an APC-depleted mitotic HeLa cell expressing EB1-GFP and H2BTagRFP collected by lattice light-sheet microscopy at $10 \mathrm{~s}$ intervals are represented in a threedimensional space. Time scale: h.m.s. 


\section{Movie 3}

3D time-lapse images of control and APC-depleted mitotic HeLa cells shown in Movie 1 and Movie 2 are projected onto a 2D plane and combined for comparison. Time scale: h.m.s.

\section{Movie 4}

Representative phase contrast time-lapse videos of MCF10A, APC883, APC883+AURKA(T288D) and APC883+ $\beta$-catenin KD\#1 (shRNA\#1) cells collected at 10 min intervals for 64 hrs. Time scale: h.m.s.

\section{Movie 5}

Representative phase contrast time-lapse videos of MCF10A, APC883, near-tetraploid APC883 cells that spontaneously appeared after $>5$ months of passaging, and APC883+TPX2 cells collected at $10 \mathrm{~min}$ intervals for $64 \mathrm{hrs}$. The MCF10A and APC883 videos are the same as those shown in Movie 4. Time scale: h.m.s.

\section{Movie 6}

Representative phase contrast time-lapse videos of MCF10A, APC883, MCF10A+MM $\beta$ catenin and APC883+ $\beta$-catenin KD\#2 (shRNA\#2) cells collected at 10 min intervals for 64 hrs. The MCF10A and APC883 videos are the same as those shown in Movie 4. Time scale: h.m.s. 


\section{Table 1}

MCF10A

\begin{tabular}{|c|c|c|}
\hline $\begin{array}{l}\text { Type } \\
\text { No. }\end{array}$ & Karyotype & $\begin{array}{l}\text { Numbुe } \\
\text { of cells. }\end{array}$ \\
\hline 1 & $48, X X, \operatorname{del}(1)(q 11 q 32),+i(1)(q 10), \operatorname{del}(3)(p 21), \operatorname{dup}(8)(p ?), \operatorname{add}(9)(p 24),+20$ & $10 \stackrel{0}{0}$ \\
\hline 2 & $47, \mathrm{XX}, \operatorname{del}(1)(q 11 q 32),+\mathrm{i}(1)(q 10), \operatorname{del}(3)(\mathrm{p} 21), \operatorname{dup}(8)(\mathrm{p} ?), \operatorname{add}(9)(\mathrm{p} 24)$ & \\
\hline 3 & 46,XX,del(1)(q11q32), +i(1)(q10), del(3)(p21), der(5)ins(5)(q10?)inv(5)(q11.2q35), dup(8)(p?), add(9)(p24),-21 & $\frac{10}{2}$ \\
\hline 4 & $47, \mathrm{XX}, \operatorname{del}(1)(\mathrm{q} 11 \mathrm{q} 32),+\mathrm{i}(1)(\mathrm{q} 10), \operatorname{del}(3)(\mathrm{p} 21), \operatorname{dup}(8)(\mathrm{p} ?), \operatorname{add}(9)(\mathrm{p} 24),+20,-21$ & 10 \\
\hline 5 & $47, \mathrm{XX}, \operatorname{del}(1)(\mathrm{q} 11 \mathrm{q} 32),+\mathrm{i}(1)(\mathrm{q} 10), \operatorname{del}(3)(\mathrm{p} 21), \operatorname{dup}(8)(\mathrm{p} ?), \operatorname{add}(9)(\mathrm{p} 24),-19,+20$ & \\
\hline 6 & $48, X X, \operatorname{del}(1)(q 11 q 32),+i(1)(q 10), \operatorname{del}(3)(p 21),+\operatorname{dup}(8)(p ?)$ & \\
\hline 7 & 49,XX,del(1)(q11q32),+i(1)(q10),del(3)(p21),dup(8)(p?),+dup(8)(p?),add(9)(p24),+del(20)(q10) & \\
\hline
\end{tabular}

Xel(1)(q11q32) +i(1)(q10), del(3)(p21),der(5)ins(5)(q10?)inv(5)(q11.2q35),dup(8)(p?), add(9)(p24),-21

$4 \quad 47, X X, \operatorname{del}(1)(q 11 q 32)+i(1)(q 10), \operatorname{del}(3)(p 21), \operatorname{dup}(8)(p ?), \operatorname{add}(9)(p 24)+20,-21$

$6 \quad 48, X X, \operatorname{del}(1)(q 11 q 32),+i(1)(q 10), \operatorname{del}(3)(p 21),+\operatorname{dup}(8)(p ?)$

7 49,XX,del(1)(q11q32),+i(1)(q10),del(3)(p21),dup(8)(p?),+dup(8)(p?), add(9)(p24),+del(20)(q10) 
APC883

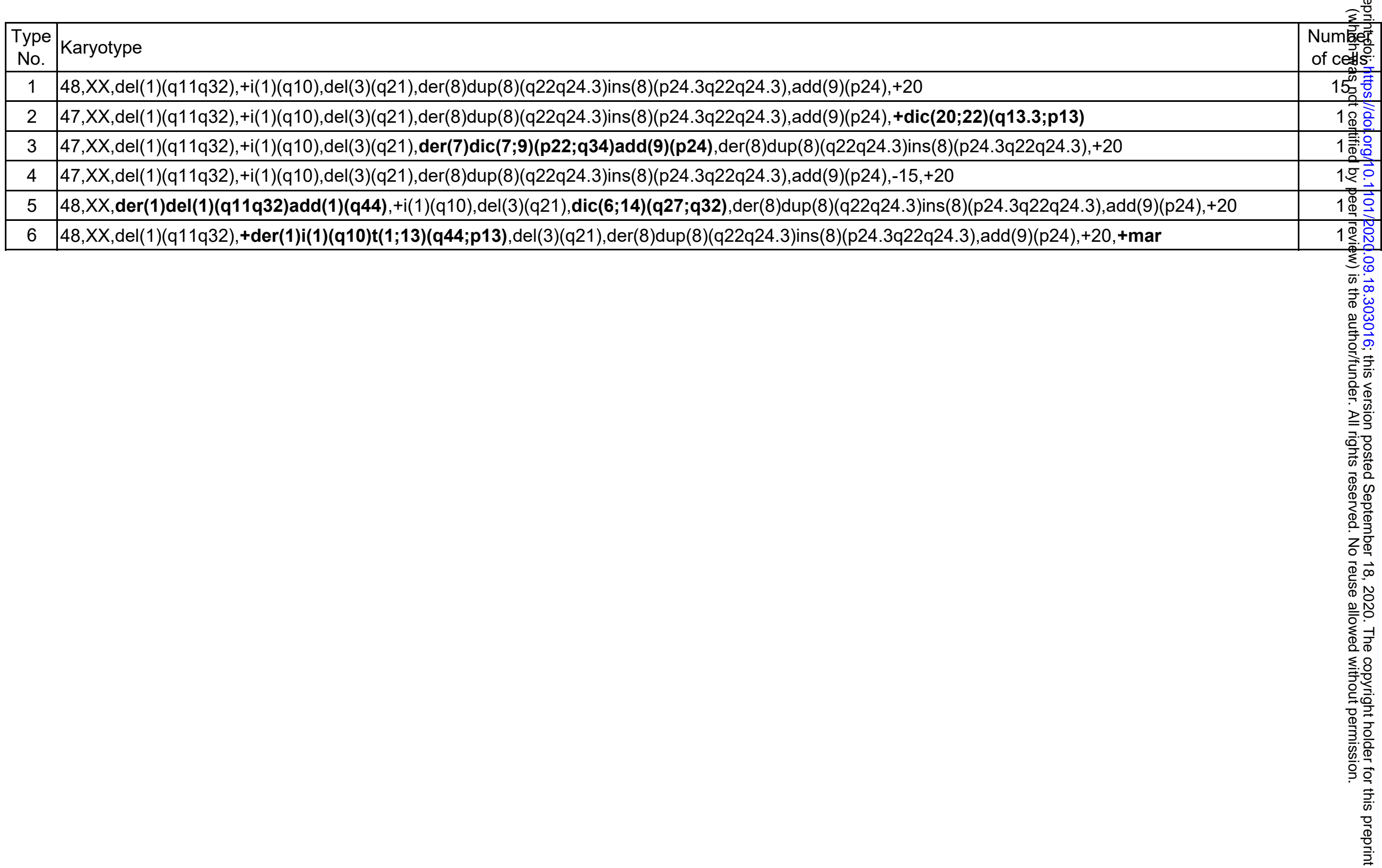


APC883 + $\beta$-catenin shRNA\#1

\begin{tabular}{|c|c|c|}
\hline $\begin{array}{l}\text { Type } \\
\text { No. }\end{array}$ & Karyotype & 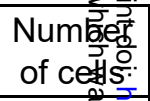 \\
\hline 1 & $48, X X, \operatorname{del}(1)(q 11 q 32),+i(1)(q 10), \operatorname{del}(3)(q 21), \operatorname{der}(8) \operatorname{dup}(8)(q 22 q 24.3)$ ins(8)(p24.3q22q24.3), add(9)(p24),+20 & $15_{5}^{\circ} \bar{c}$ \\
\hline 2 & 47,XX,del(1)(q11q32),+i(1)(q10), del(3)(q21), der(8)dup(8)(q22q24.3)ins(8)(p24.3q22q24.3), add(9)(p24),+20,-21 & $3 \ddot{\circ} \frac{0}{0}$ \\
\hline 3 & 47,XX,del(1)(q11q32),+i(1)(q10), del(3)(q21), der(8)dup(8)(q22q24.3)ins(8)(p24.3q22q24.3),add(9)(p24),+20,-22 & 1 言浐 \\
\hline 4 & 48,XX,del(1)(q11q32),+i(1)(q10), del(3)(q21), der(8)dup(8)(q22q24.3)ins(8)(p24.3q22q24.3),add(9)(p24),-18,+20,+mar & $10 \overrightarrow{0}$ \\
\hline
\end{tabular}


APC883 + $\beta$-catenin shRNA\#2

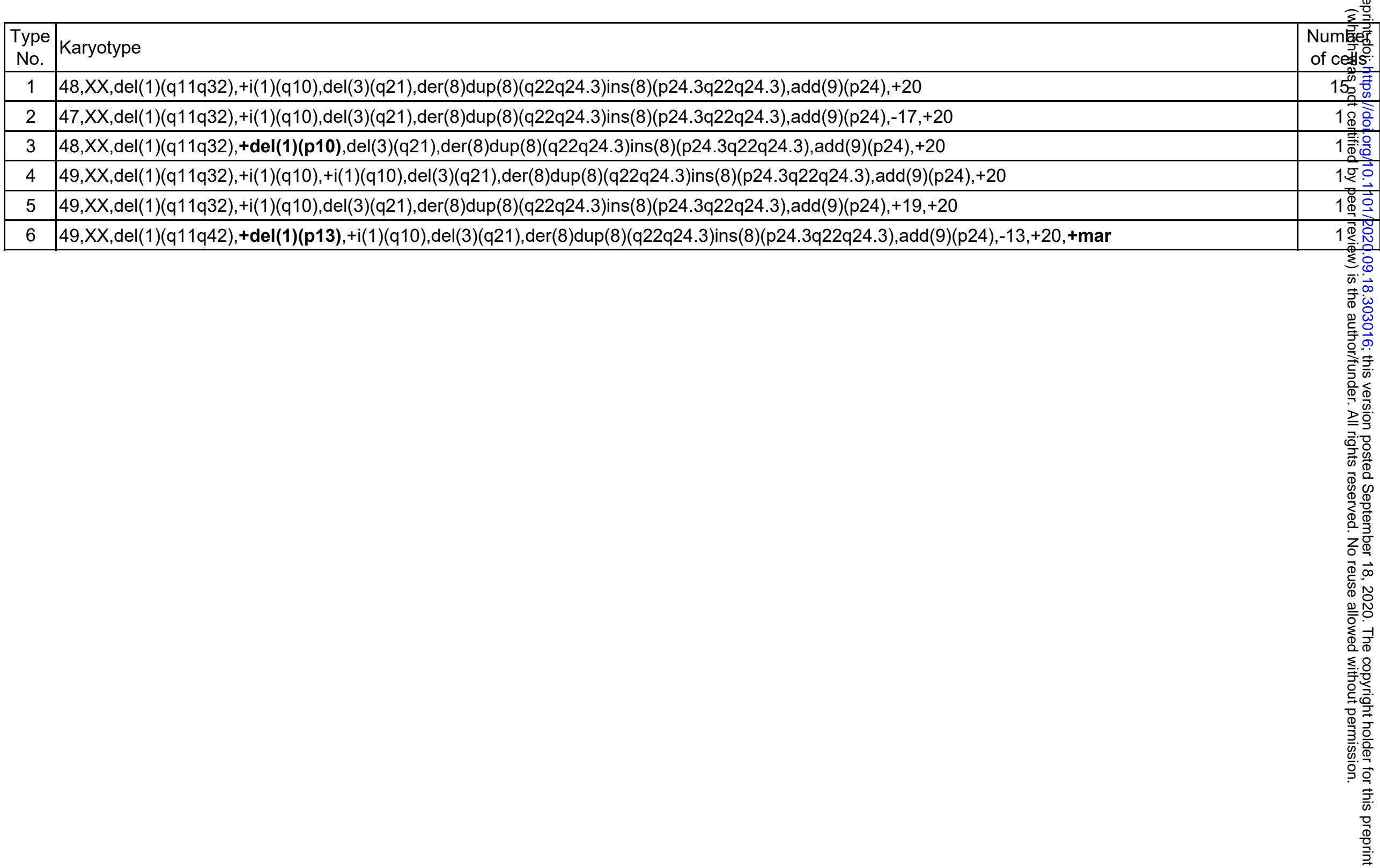


APC883 + AURKA(T288D)

\begin{tabular}{|c|c|c|}
\hline $\begin{array}{l}\text { Type } \\
\text { No. }\end{array}$ & Karyotype & $\begin{array}{l}\text { Number } \\
\text { of ce过s }\end{array}$ \\
\hline 1 & $48, X X, \operatorname{del}(1)(q 11 q 32),+i(1)(q 10), \operatorname{del}(3)(q 21), \operatorname{der}(8) \operatorname{dup}(8)(q 22 q 24.3) \operatorname{ins}(8)(p 24.3 q 22 q 24.3), \operatorname{add}(9)(p 24),+20$ & 18 \\
\hline 2 & 47,XX,del(1)(q11q32),+i(1)(q10),del(3)(q21),der(8)dup(8)(q22q24.3)ins(8)(p24.3q22q24.3),add(9)(p24) & \\
\hline 3 & 47,XX,del(1)(q11q32),+i(1)(q10), del(3)(q21),der(8)dup(8)(q22q24.3)ins(8)(p24.3q22q24.3), add(9)(p24),-14,+20 & \\
\hline
\end{tabular}


APC883 + TPX2

\begin{tabular}{|c|c|c|}
\hline $\begin{array}{c}\text { Type } \\
\text { No. }\end{array}$ & Karyotype & $\begin{array}{l}\text { Number } \\
\text { of ceis: }\end{array}$ \\
\hline 1 & $48, X X, \operatorname{del}(1)(q 11 q 32),+i(1)(q 10)$, del(3)(q21), der(8)dup(8)(q22q24.3)ins(8)(p24.3q22q24.3), add(9)(p24),+20 & 16 के \\
\hline 2 & $47, \mathrm{XX}, \mathrm{del}(1)(\mathrm{q} 11 \mathrm{q} 32),+\mathrm{i}(1)(\mathrm{q} 10), \mathrm{del}(3)(\mathrm{q} 21), \mathrm{der}(8) \mathrm{dup}(8)(\mathrm{q} 22 \mathrm{q} 24.3) \mathrm{ins}(8)(\mathrm{p} 24.3 q 22 q 24.3), \operatorname{add}(9)(\mathrm{p} 24),-14,+\operatorname{mar}$ & $1 \underset{\mathbb{\Phi}}{10}$ \\
\hline 3 & $47, \mathrm{XX}, \mathrm{del}(1)(\mathrm{q} 11 \mathrm{q} 32),+\mathrm{i}(1)(\mathrm{q} 10), \mathrm{del}(3)(\mathrm{q} 21), \operatorname{der}(8) \operatorname{dup}(8)(\mathrm{q} 22 \mathrm{q} 24.3) \operatorname{ins}(8)(\mathrm{p} 24.3 q 22 q 24.3) \operatorname{add}(8)(\mathrm{q} 24.3), \operatorname{add}(9)(\mathrm{p} 24),-15,+20$ & 1 害兑 \\
\hline 4 & 47,XX,del(1)(q11q32),+i(1)(q10), del(3)(q21),dic(4;22)(q35;p12), der(8)dup(8)(q22q24.3)ins(8)(p24.3q22q24.3),add(9)(p24),+20 & $1 \overrightarrow{0} \overrightarrow{0}$ \\
\hline 5 & 48,XX,der(1)del(1)(q11q32)t(1;12)(p36.3;q13),+i(1)(q10), del(3)(q21), der(8)dup(8)(q22q24.3)ins(8)(p24.3q22q24.3),add(9)(p24),+20 & 1离官 \\
\hline
\end{tabular}




\section{Near-tetraploid APC883}

\begin{tabular}{|c|c|c|}
\hline \begin{tabular}{l|l} 
Type \\
No.
\end{tabular} & Karyotype & 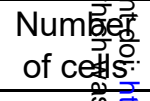 \\
\hline 1 & $\begin{array}{l}\text { 95,XXXX, del(1)(q11q32), del(1)(q11q32),+i(1)(q10), del(3)(p21), del(3)(p21),-6, der(8)dup(8)(q22q24.3)ins(8)(p24.3q22q24.3), } \\
\operatorname{der}(8) \operatorname{dup}(8)(q 22 q 24.3) \operatorname{ins}(8)(p 24.3 q 22 q 24.3),+\operatorname{der}(8) \operatorname{dup}(8)(q 22 q 24.3) i n s(8)(p 24.3 q 22 q 24.3), \operatorname{add}(9)(p 24), \operatorname{add}(9)(p 24),+20,+20\end{array}$ & 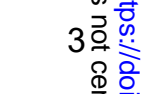 \\
\hline 2 & $\begin{array}{l}\text { 96,XXXX, del(1)(q11q32), del(1)(q11q32),+i(1)(q10),+i(1)(q10), del(3)(p21), del(3)(p21),-6, der(8)dup(8)(q22q24.3)ins (8)(p24.3q22q24.3), } \\
\operatorname{der}(8) \operatorname{dup}(8)(q 22 q 24.3) i n s(8)(p 24.3 q 22 q 24.3),+\operatorname{der}(8) \operatorname{dup}(8)(q 22 q 24.3) i n s(8)(p 24.3 q 22 q 24.3), \operatorname{add}(9)(p 24), \operatorname{add}(9)(p 24),+20,+20\end{array}$ & $3 \stackrel{\overrightarrow{\frac{\vec{D}}{2}}}{\underline{0}}$ \\
\hline 3 & $\begin{array}{l}\text { 94,XXXX, del(1)(q11q32), del(1)(q11q32),+i(1)(q10), del(3)(p21), del(3)(p21),-6, der(8)dup(8)(q22q24.3)ins(8)(p24.3q22q24.3), } \\
\operatorname{der}(8) \operatorname{dup}(8)(q 22 q 24.3) \operatorname{ins}(8)(p 24.3 q 22 q 24.3),+\operatorname{der}(8) \operatorname{dup}(8)(q 22 q 24.3) \text { ins }(8)(p 24.3 q 22 q 24.3), \text { add }(9)(p 24), \text { add }(9)(p 24),-13,+20,+20\end{array}$ & \\
\hline 4 & $\begin{array}{l}\text { 93,XXXX, del(1)(q11q32), del(1)(q11q32),+i(1)(q10), del(3)(p21), del(3)(p21),-6, der(8)dup(8)(q22q24.3)ins(8)(p24.3q22q24.3), } \\
\operatorname{der}(8) \operatorname{dup}(8)(q 22 q 24.3) \operatorname{ins}(8)(p 24.3 q 22 q 24.3),+\operatorname{der}(8) \operatorname{dup}(8)(q 22 q 24.3) i n s(8)(p 24.3 q 22 q 24.3), \operatorname{add}(9)(p 24), \operatorname{add}(9)(p 24),-15,-18,+20,+20\end{array}$ & $\begin{array}{r}1 \bar{\omega} \\
\text { 产 }\end{array}$ \\
\hline 5 & $\begin{array}{l}\text { 93,XXXX, del(1)(q11q32), del(1)(q11q32),i(1)(q10),-2, del(3)(p21), del(3)(p21),-6, der(8)dup(8)(q22q24.3)ins(8)(p24.3q22q24.3), } \\
\text { der(8)dup(8)(q22q24.3)ins(8)(p24.3q22q24.3),+der(8)dup(8)(q22q24.3)ins(8)(p24.3q22q24.3), add(9)(p24),add(9)(p24),+20,+20 }\end{array}$ & \\
\hline 6 & $\begin{array}{l}\text { 94,XXXX, del(1)(q11q32), del(1)(p10),+i(1)(q10), del(3)(p21),-3,-6, der(8)dup(8)(q22q24.3)ins(8)(p24.3q22q24.3), } \\
\operatorname{der}(8) \operatorname{dup}(8)(q 22 q 24.3) \operatorname{ins}(8)(p 24.3 q 22 q 24.3),+\operatorname{der}(8) \operatorname{dup}(8)(q 22 q 24.3) i n s(8)(p 24.3 q 22 q 24.3), \operatorname{add}(9)(p 24), \operatorname{add}(9)(p 24),+20,+20\end{array}$ & $1 \stackrel{\stackrel{?}{\stackrel{Q}{Q}}}{\geqq}$ \\
\hline 7 & $\begin{array}{l}\text { 94,XXXX, del(1)(q11q32), del(1)(q11q32),i(1)(q10), del(3)(p21),del(3)(p21),-6, der(8)dup(8)(q22q24.3)ins(8)(p24.3q22q24.3), } \\
\text { der(8)dup(8)(q22q24.3)ins(8)(p24.3q22q24.3),+der(8)dup(8)(q22q24.3)ins(8)(p24.3q22q24.3), add(9)(p24),add(9)(p24),+20,+20 }\end{array}$ & \\
\hline 8 & $\begin{array}{l}\text { 94,XXXX, del(1)(q11q32), del(1)q11q32),+i(1)(q10), del(3)(p21), del(3)(p21),-6, der(8)dup(8)(q22q24.3)ins(8)(p24.3q22q24.3), } \\
\operatorname{der}(8) \operatorname{dup}(8)(q 22 q 24.3) i n s(8)(p 24.3 q 22 q 24.3),+\operatorname{der}(8) \operatorname{dup}(8)(q 22 q 24.3) i n s(8)(p 24.3 q 22 q 24.3), \operatorname{add}(9)(p 24), \operatorname{add}(9)(p 24), \operatorname{add}(11)(p 15),+20,+20,-21\end{array}$ & \\
\hline 9 & $\begin{array}{l}\text { 95,XXXX, del(1)(q11q32), del(1)(q11q32),+i(1)(q10),+i(1)(q10), del(3)(p21), del(3)(p21),-6, der(8)dup(8)(q22q24.3)ins(8)(p24.3q22q24.3), } \\
\operatorname{der}(8) \operatorname{dup}(8)(q 22 q 24.3) \operatorname{ins}(8)(p 24.3 q 22 q 24.3),+\operatorname{der}(8) \operatorname{dup}(8)(q 22 q 24.3) i n s(8)(p 24.3 q 22 q 24.3), \operatorname{add}(9)(p 24), \text { add }(9)(p 24),-13,+20,+20\end{array}$ & \\
\hline 10 & $\begin{array}{l}\text { 95,XXXX, del(1)(q11q32), del(1)(q11q32),i(1)(q10),+i(1)(q10), del(3)(p21), del(3)(p21),-6, der(8)dup(8)(q22q24.3)ins(8)(p24.3q22q24.3), } \\
\operatorname{der}(8) \operatorname{dup}(8)(q 22 q 24.3) i n s(8)(p 24.3 q 22 q 24.3),+\operatorname{der}(8) \operatorname{dup}(8)(q 22 q 24.3) \operatorname{ins}(8)(p 24.3 q 22 q 24.3), \operatorname{add}(9)(p 24), \operatorname{add}(9)(p 24),+20,+20\end{array}$ & \\
\hline 11 & $\begin{array}{l}\text { 96,XXXX, del(1)(q11q32), del(1)(q11q32),+i(1)(q10),+i(1)(q10), del(3)(p21), del(3)(p21),-6, der(8)dup(8)(q22q24.3)ins(8)(p24.3q22q24.3), } \\
\operatorname{der}(8) \operatorname{dup}(8)(q 22 q 24.3) i n s(8)(p 24.3 q 22 q 24.3),+\operatorname{der}(8) \operatorname{dup}(8)(q 22 q 24.3) i n s(8)(p 24.3 q 22 q 24.3),+i(8)(q 10), \operatorname{add}(9)(p 24), \operatorname{add}(9)(p 24),-19,+20,+20\end{array}$ & \\
\hline 12 & $\begin{array}{l}\text { 96,XXXX, del(1)(q11q32), del(1)(q11q32),+i(1)(q10),+i(1)(q10), del(3)(p21), del(3)(p21),-6, der(8)dup(8)(q22q24.3)ins(8)(p24.3q22q24.3), } \\
\operatorname{der}(8) \operatorname{dup}(8)(q 22 q 24.3) \text { ins(8)(p24.3q22q24.3), +der(8)dup(8)(q22q24.3)ins(8)(p24.3q22q24.3), add(9)(p24), add(9)(p24),add(13)(p13),+20,+20 }\end{array}$ & \\
\hline 12 & $97, X X X X, \operatorname{del}(1)(q 11 q 32), \operatorname{del}(1)(q 11 q 32),+\operatorname{del}(1)(p 10),+i(1)(q 10),+i(1)(q 10), \operatorname{del}(3)(p 21), \operatorname{del}(3)(p 21),-6, \operatorname{der}(8) \operatorname{dup}(8)(q 22 q 24.3) i n s(8)(p 24.3 q 22 q 24.3)$ & 1 \\
\hline
\end{tabular}




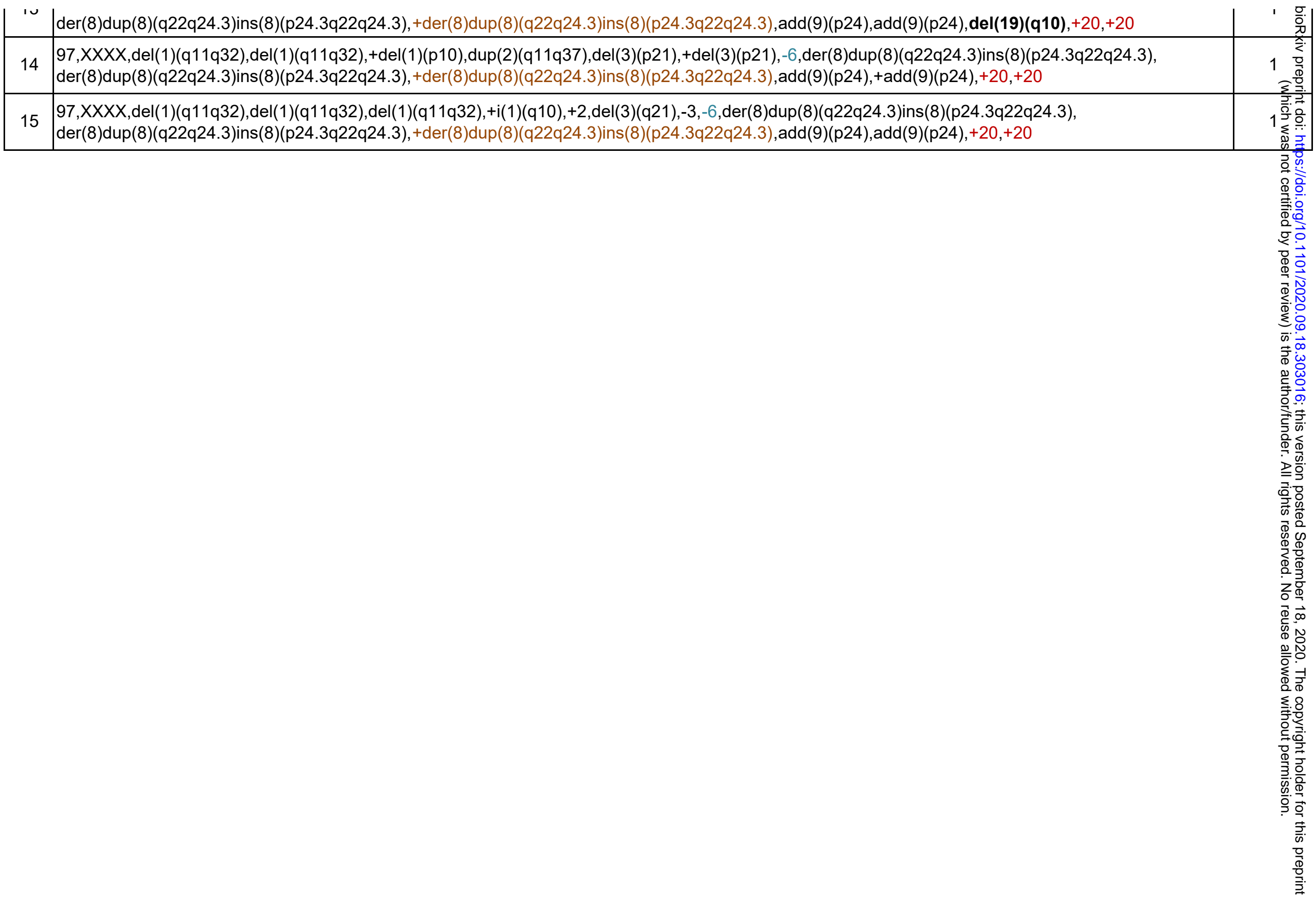

